Original paper

\title{
Initial replacement stage of primary uranium (U'v) minerals by supergene alteration: association of uranyl-oxide hydroxy-hydrates and "calciolepersonnite" from the Krátka Dolina Valley (Gemerská Poloma, Gemeric Unit, Western Carpathians, Slovakia)
}

\author{
Štefan FERENC ${ }^{*}$, Adrián BIROŇ², Tomáš MIKUŠ2, Ján SPIŠIAK¹, Šimon BUDZÁK \\ ${ }^{1}$ Department of Geography and Geology, Faculty of Natural Sciences, Matej Bel University, Tajovského 40, 97401 Banská Bystrica, \\ Slovak Republic; stefan.ferenc@umb.sk \\ ${ }^{2}$ Earth Science Institute of the Slovak Academy of Sciences, Ďumbierska 1, 97411 Banská Bystrica, Slovak Republic \\ ${ }^{3}$ Department of Chemistry, Faculty of Natural Sciences, Matej Bel University, Tajovského 40, 97401 Banská Bystrica, Slovak Republic \\ * Corresponding author
}

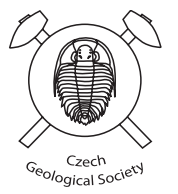

Mineral association with uranyl-oxide hydroxy-hydrates and uranyl carbonate-silicates was found in J-1 quartz vein containing U-Au mineralization at Krátka Dolina Valley (Rožňava district, Slovakia). The vein penetrated Lower Palaeozoic graphitic phyllites and metalydites (Vlachovo Fm., Gemeric Unit).

Among minerals identified at the site, becquerelite I is characterized by the highest Ca content and its composition is close to the ideal. On the other hand, becquerelite II is characterized by increased $\mathrm{K}$ at the expense of $\mathrm{Ca}$. The average chemical composition of both types of becquerelite can be expressed by the empirical formulae: $\left(\mathrm{Ca}_{0.85} \mathrm{~K}_{0.04} \mathrm{Na}_{0.01} \mathrm{Fe}_{0.02} \mathrm{Zn}_{0.02} \mathrm{Ba}_{0.01}\right.$ $\left.\mathrm{Pb}_{0.01}\right)_{\Sigma 0.96}\left[\left(\mathrm{UO}_{2}\right)_{6} \mathrm{O}_{4}(\mathrm{OH})_{5.84}\right] \cdot 8 \mathrm{H}_{2} \mathrm{O}$ (becquerelite I) and $\left(\mathrm{Ca}_{0.36} \mathrm{~K}_{0.27} \mathrm{Na}_{0.01} \mathrm{Fe}_{0.02} \mathrm{Zn}_{0.02} \mathrm{~Pb}_{0.01} \mathrm{Bi}_{0.01}\right)_{\Sigma 0.78}\left[\left(\mathrm{UO}_{2}\right)_{6} \mathrm{O}_{4}(\mathrm{OH})_{5.29}\right] \cdot 8 \mathrm{H}_{2} \mathrm{O}$ (becquerelite II). Vandendriesscheite with unusual chemical composition imitates a transition phase between gauthierite and vandendriesscheite. Negative correlation of $\mathrm{K}$ vs. $\mathrm{Pb}$ indicates that in the studied mineral phase lead is partially replaced by potassium (and other cations). An average chemical composition of the studied vandendriesscheite can be expressed as: $\left(\mathrm{K}_{0.49} \mathrm{Na}_{0.02}\right)_{\Sigma 0.51}\left(\mathrm{~Pb}_{1.20} \mathrm{Fe}_{0.05} \mathrm{Zn}_{0.04} \mathrm{Ba}_{0.03} \mathrm{Sr}_{0.02} \mathrm{Al}_{0.02}\right)_{\Sigma 1.36}\left[\left(\mathrm{UO}_{2}\right)_{10} \mathrm{O}_{6}\left(\mathrm{SiO}_{4}\right)_{0.05}\left(\mathrm{PO}_{4}\right)_{0.02}(\mathrm{OH})_{10.86}\right] \cdot 11 \mathrm{H}_{2} \mathrm{O}$. A leesite-like phase, with an average composition $\left(\mathrm{K}_{0.72} \mathrm{Sr}_{0.01} \mathrm{Ba}_{0.02} \mathrm{Fe}_{0.03} \mathrm{Zn}_{0.01} \mathrm{~Pb}_{0.02} \mathrm{Al}_{0.02}\right)_{\Sigma 0.83}\left(\mathrm{H}_{2} \mathrm{O}\right)_{2}\left[\left(\mathrm{UO}_{2}\right)_{4} \mathrm{O}_{2}\left(\mathrm{SiO}_{4}\right)_{0.01}(\mathrm{OH})_{5,00}\right]_{\Sigma 11,01}$ $\cdot 3 \mathrm{H}_{2} \mathrm{O}$, was found only rarely. An unnamed mineral phase, with chemical composition close to lepersonnite-(Gd), designated as "calciolepersonnite", is younger at the studied site then uranyl-oxide hydroxy-hydrates. Compared to the ideal lepersonnite- $(\mathrm{Gd})$ formula, there is a lower REE content at the cationic position, an increased $\mathrm{Ca}$ and there are also monovalent cations (especially $\mathrm{K}$ ) entering the structure. An average "calciolepersonnite" chemical composition is: $\left(\mathrm{K}_{0.62} \mathrm{Na}_{0.09}\right)_{\Sigma 0.71}\left(\mathrm{Ca}_{2.08} \mathrm{Mg}_{0.04} \mathrm{Sr}_{0.02} \mathrm{Ba}_{0.02} \mathrm{Fe}_{0.05} \mathrm{Zn}_{0.05} \mathrm{~Pb}_{0.03}\right)_{\Sigma 2.30}(\mathrm{Y}+\mathrm{REE})_{\Sigma 0.92}\left[\left(\mathrm{UO}_{2}\right)_{23.76}\left\{\left(\mathrm{SiO}_{4}\right)_{3.19}\left(\mathrm{PO}_{4}\right)_{0.11}\left(\mathrm{AsO}_{4}\right)_{0.02}\left(\mathrm{SO}_{4}\right)_{0.02}\right\}_{\Sigma 3.34}\right.$ $\left.\left(\mathrm{CO}_{3}\right)_{8}(\mathrm{OH})_{26.37}\right] \cdot 46.82 \mathrm{H}_{2} \mathrm{O}$.

In the supergene zone of J-1 vein at Gemerská Poloma, three stages of development can be defined: (I) formation of uranyl-oxide hydroxy-hydrates that directly, partially or completely, replace $\mathrm{U}^{\mathrm{IV}}$ minerals; (II) formation of uranyl carbonate-silicates (i.e., "calciolepersonnite") that replace uranyl-oxide hydroxy-hydrates, apparently indicating shift to relative acid environment (but still remaining alkaline to neutral $\mathrm{pH}$ ) and (III) formation of uranyl phosphates/arsenates of the autunite group ("uranium micas") that precipitated relatively far from accumulations of primary ( $\mathrm{U}^{\mathrm{IV}}$ ) minerals (in cracks and cavities of the gangue, or in the surrounding non-mineralized rocks). Their origin documents the change of alkaline-neutral to acidic environment, due the more advanced weathering of vein sulphides. Given the absence of $\mathrm{Y}+\mathrm{REE}$ in older uranyl-oxide hydroxy-hydrates that directly replace brannerite, most of these elements required for "calciolepersonnite" formation were probably released from the host rocks and not from the primary, hydrothermal uranium minerals.

Keywords: uranyl-oxide hydroxy-hydrates, lepersonnite-(Gd), brannerite oxidation, uranyl minerals, Gemeric Unit, Western Carpathians Received: 1 March, 2018; accepted: 24 August, 2018; handling editor: J. Sejkora

\section{Introduction}

Uranium mineralization is common in almost all of the main tectonic units of the Western Carpathians, but the economic parameters reaches only in the Gemeric, and less so in the Hronic and Tatric units. Uraninite is the dominant primary mineral at all occurrences of $U$ ores.
Common uraninite guides are brannerite and coffinite, whereby the latter is known mainly from the U-Mo deposits in the Gemeric Unit (Košice-Kurišková, Novoveská Huta). Brannerite is a dominant at the occurrence Petrova Hora Mts. near Krompachy (Gemeric Unit), and common in Kálnica and Selec deposits in the Tatric Unit (Rojkovič 1997). It also occurs in the hydrothermal 

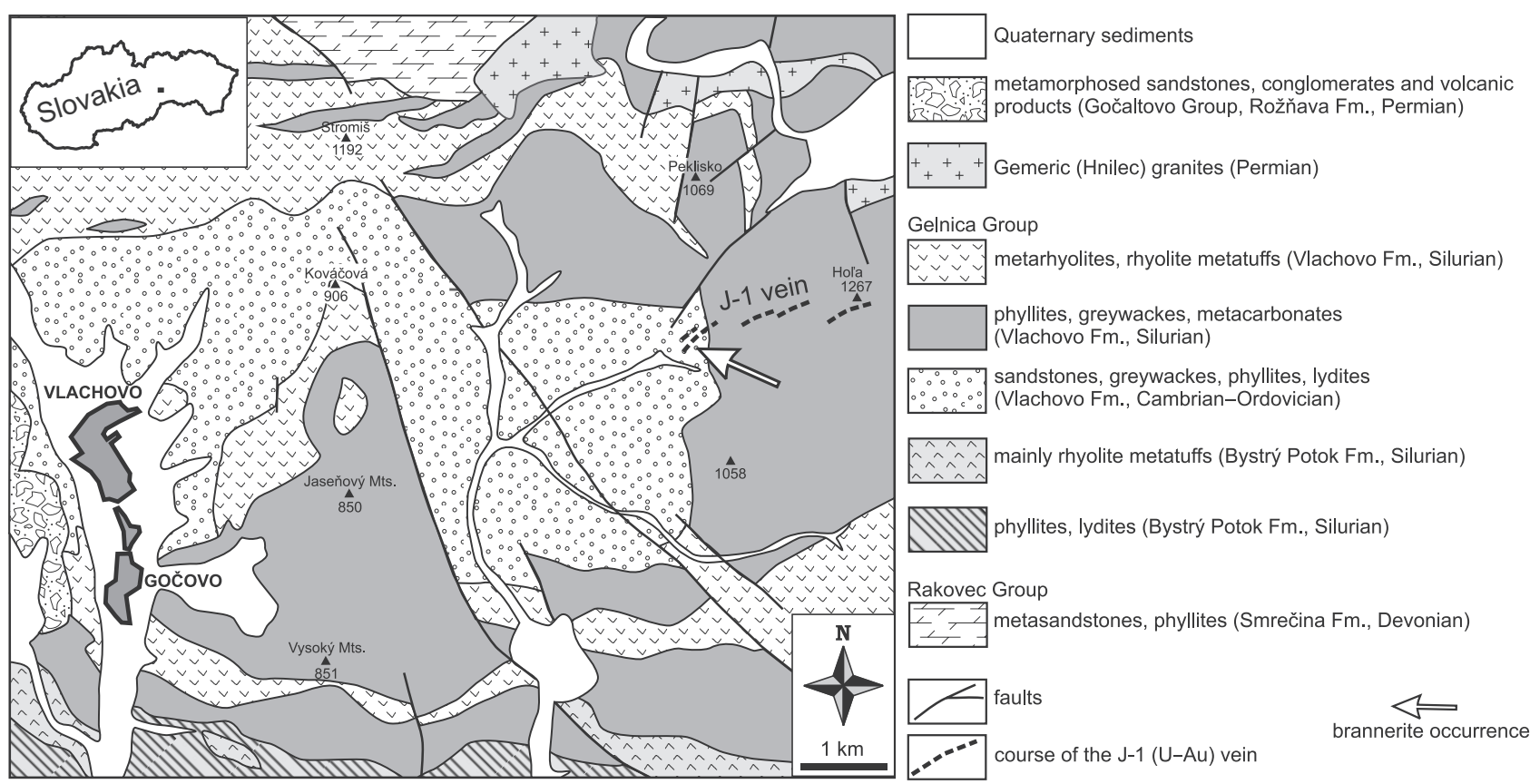

Fig. 1 Simplified geological map of the J-1 vein surroundings (according to Bajaník et al. 1984).

systems connected with the Cretaceous Rochovce granite intrusion (Poller et al. 2001; Kohút et al. 2013). The most interesting occurrences of brannerite represent Gemeric quartz ( \pm apatite) veins that contain U-REE mineralization in the Krátka Dolina Valley (near Gemerská Poloma), Peklisko (at the Hnilec village), and at the end of the Gelnická Dolina Valley (Zimná Voda occurrence) near Prakovce (Novotný and Čížek 1979; Rojkovič and Novotný 1993; Rojkovič et al. 1997; Novotný et al. 1999; Donát et al. 2000).

Primary uranium $\left(\mathrm{U}^{\mathrm{IV}}\right)$ minerals occurring on Slovak $\mathrm{U}$ mineralizations are mostly very fine-grained only. For this reason, it is difficult to observe the initial stage of their oxidation - in-situ replacing by mixed $\left(\mathrm{U}^{\mathrm{IV}}\right)-\left(\mathrm{U}^{\mathrm{VI}}\right)$ minerals or by uranyl-oxide hydroxy-hydrates. Only the veins with U-REE mineralization in the Gemeric Unit (Krátka Dolina, Peklisko, Zimná voda) provided macroscopic samples of uraninite and brannerite (usually up to $1 \mathrm{~cm}$, rarely up to $10 \mathrm{~cm}$ in size) that are often replaced by markedly yellow uranyl minerals, forming partial to full pseudomorphs. At the Gemerská Poloma-Krátka Dolina occurrence, a quartz gangue with massive accumulations of brannerite was found in the debris material. The present contribution deals with the mineralogical characteristics of supergene uranyl minerals that partially replace the massive, primary ore.

\section{Geological setting}

The Krátka Dolina U-Au vein mineralization occurrence is located in the Gelnica Group of the Gemeric Unit.
This Unit consists of a system of north-vergent nappes composed mainly of metamorphosed pre-Carboniferous complexes and Upper Palaeozoic to Lower Triassic syn- and post-orogenic formations (e.g. Mahel' and Vozár 1971; Németh et al. 2000; Radvanec and Grecula 2016). Most often it is divided into the Northern (Klátov, Rakovec, Črmel' and Ochtiná groups) and Southern (Gelnica and Štós groups) parts. The Gelnica Group is represented by a several thousand meters thick Palaeozoic volcanogenic (rhyolite-dacite) flysch (Snopko and Ivanička 1978; Ivanička et al. 1989), and its origin was associated with an active margin of Gondwana (Vozárová 1993; Putiš et al. 2008). Both sedimentary and volcanic rocks have undergone regional metamorphism under the chlorite zone of the greenschist-facies conditions (Faryad 1991a, b). The Gelnica Group (from the bottom to top) is divided into Vlachovo, Bystrý Potok and Drnava formations (sensu Bajaník et al. 1983, 1984; Ivanička et al. 1989) intruded by the Permian Gemeric granites. Although according to geological maps the Bystrý Potok $\mathrm{Fm}$. is stratigraphically assigned to the Silurian, its magmatic rocks yielded Middle-Late Ordovician U-Th- $\mathrm{Pb}$ SHRIMP zircon ages of $465.8 \pm 1.5 \mathrm{Ma}$ (Putiš et al. 2008; Vozárová et al. 2010), 460-465 Ma respectively (Vozárová et al. 2017).

Vein $\mathrm{J}-1$ with $\mathrm{U}-\mathrm{Au}$ mineralization is located c. $15 \mathrm{~km}$ $\mathrm{N}$ of the district town Rožn̆ava, on the south-facing slope of Krátka Dolina Valley. Massive brannerite was found in the eastern part of the vein, $8.6 \mathrm{~km}$ to the NNE from Gemerská Poloma village, $7 \mathrm{~km}$ to the ENE from Vlachovo village, $2 \mathrm{~km}$ to the WSW from the Hol'a Mts. (1267 m a. s. 1.) at an altitude of about $700 \mathrm{~m}$ a. s. 1. Geographical 
coordinates of brannerite finding are $\mathrm{N} 48^{\circ} 47^{\prime} 14.9^{\prime \prime}$ and E 2030'21.5".

The surroundings of occurrence are built mainly by the Vlachovo Fm. (Cambrian-Silurian; Fig. 1). It is formed by metarhyolites and rhyolite metatuffs; metasediments are represented mainly by phyllites (sericite, quartz-sericite, chlorite-sericite and sericite-graphite); metamorphosed greywackes, sandstones, conglomerates, carbonates, and lydites are also present. According to the recent concept of the Gemeric Unit (Grecula et al. 2009, 2011) were rocks of the Vlachovo Fm. divided into Betliar and Smolník formations (Ordovician-Devonian).

The J-1 vein consists of discontinuous structures (total length of $2.25 \mathrm{~km}$ ) cutting the graphitic phyllites and metalydites of the Vlachovo Fm. (Donát et al. 2000). The individual segments have a length of 150-400 $\mathrm{m}$, strike WSW-ENE to SW-NE and dip $38-60^{\circ}$ to the SE. The vein has a lenticular character and very variable thickness $(0.01-1 \mathrm{~m})$. Dominant vein mineral is quartz, containing brannerite, uraninite, gold, pyrite, galena, chalcopyrite, bornite, pyrrhotite, arsenopyrite, marcasite, sphalerite, glaucodote, gersdorffite, bismuthinite, millerite, bravoite, rutile, apatite, chlorite and sericite. The supergene zone is characterized by the occurrence of goethite, fourmarierite, autunite and torbernite/metatorbernite (Varček 1977; Rojkovič and Novotný 1993; Ferenc et al. 2003). Uranium content in the gangue ranges from 0.08 to $6.57 \mathrm{wt}$ \% (predominantly $0.1-0.88$ wt. \%), the maximum determined $\mathrm{Au}$ concentration was 17.2 ppm (Donát et al. 2000).

There are two fundamentally different views on the genesis of the quartz- $( \pm$ apatite $)$ veins with U-REE $( \pm \mathrm{Au})$ mineralization in the Gemeric Unit. Rojkovič (1997) and Rojkovič et al. $(1995,1999)$ considered mineralization as Hercynian, whereby the Permian Gemeric granites presumably provided fluids and thermal energy. The hydrothermal effect of granites could have caused mobilization of P, REE and $U$ from the surrounding Lower Palaeozoic graphitic phyllites, metalydites and metaphosphates, and subsequent concentration of these elements in the hydrothermal veins. Recent electron-microprobe U-Pb uraninite dating from Čučma by Števko et al. (2014) gave a Palaeoalpine age (207 \pm 2 Ma; Late Triassic). On this basis, Permian granites are still considered as a possible source of mineralization elements. However, mobilization of P, F, U, REE and Y from granites and their deposition in the hydrothermal veins had to occur during the Late Triassic tectonothermal rejuvenation of the Gemeric Unit (Radvanec et al. 2009).

\section{Methods}

Yellow secondary uranium minerals were separated from the surface of brannerite sample to prepare pol- ished sections and mounts for powder X-ray diffraction analysis (XRD) and Infrared spectroscopic analysis (IR) respectively.

$\mathrm{X}$-ray diffraction analysis was performed on a Bruker D8 Advance equipment (Earth Science Institute of Slovak Academy of Sciences - SAS, Banská Bystrica, Slovakia) using $\mathrm{CuK}_{\alpha}(1.5418 \AA)$ radiation generated at a voltage of $40 \mathrm{kV}$ and a current of $30 \mathrm{~mA}$. The powder mount was placed in an ethanol suspension onto a $\mathrm{Si}$ single crystal. Subsequently, diffraction data were obtained under the following conditions: apertures $0.3^{\circ}-6 \mathrm{~mm}$ $-0.3^{\circ}-0.2 \mathrm{~mm}$, primary and secondary Soller aperture $2.5^{\circ}$, step $0.02^{\circ} 2 \Theta / 1.25 \mathrm{~s}$, measuring range $2.0-65.0^{\circ}$ $2 \Theta$, EDS detector Sol-XE. Diffraction patterns were evaluated using the Difrac.Eva software (Bruker AXS 2010) and PDF $2 / 2010$ database. Individual reflections were indexed according to structural data available in Downs and Hall-Wallace (2003). Unit-cell parameters were calculated from the X-ray diffraction pattern by least-squares method, using the UnitCell software (Holland and Redfern 1997).

Becquerelite was also studied by IR spectroscopy in a spectral range from 4000 to $400 \mathrm{~cm}^{-1}$, using a Nicolet iS50 equipment (Matej Bel University, Banská Bystrica, Slovakia), using conventional technique of Attenuated Total Reflection (ATR) with a synthetic diamond as a measuring crystal. During each measurement, 32 scans with step $0.482 \mathrm{~cm}^{-1}$ were taken. The two empirical equations: $\mathrm{R}_{\mathrm{U}-\mathrm{O}}=106.5\left[v_{1}\left(\mathrm{UO}_{2}\right)^{2+}\right]^{-2 / 3}+0.575 \AA$ and $\mathrm{R}_{\mathrm{U}-\mathrm{O}}=$ $91.41\left[v_{3}\left(\mathrm{UO}_{2}\right)^{2+}\right]^{-2 / 3}+0.804 \AA$ (Bartlett and Cooney 1989) were applied for the calculation of the $\mathrm{U}-\mathrm{O}$ bond lengths in uranyl ion based on observed wavenumbers.

Electron probe micro-analysis (EPMA) of mineral phases was performed by the JEOL JXA-8530F equipment (Institute of the Earth Sciences SAS, Banská Bystrica, Slovakia). Following conditions were used: accelerating voltage $15 \mathrm{kV}$, probe current $15 \mathrm{nA}$ and a beam diameter of $10 \mu \mathrm{m}$. The ZAF matrix correction was employed. The used X-ray lines, crystals, detection limits (in ppm) and natural/synthetic standards were: $\mathrm{Ca}$ $\left(\mathrm{K}_{\alpha}\right.$, PETL, 37-70) - diopside, $\mathrm{K}\left(\mathrm{K}_{\alpha}\right.$, PETL, 45-50) - orthoclase, $\mathrm{U}\left(\mathrm{M}_{\beta}\right.$, PETL, 103-116) - $\mathrm{UO}_{2}$, Th $\left(\mathrm{M}_{\alpha}\right.$, PETL, 75-79) - thorianite, $\mathrm{Pb}\left(\mathrm{M}_{\beta}\right.$, PETL, 126-173) - crocoite, $\mathrm{S}\left(\mathrm{K}_{\alpha}\right.$, PETL, 46-53) - baryte, P (K $\mathrm{K}_{\alpha}$ PETL, 68-89) apatite, $\mathrm{Y}-\left(\mathrm{L}_{\alpha}, \mathrm{PETL}, 131-166\right)-\mathrm{YPO}_{4}, \mathrm{~F}\left(\mathrm{~K}_{\alpha}, \mathrm{LDE} 1\right.$, 159-334) - fluorite, Na (K $\mathrm{K}_{\alpha}$ TAP, 68-126) - albite, Sr $\left(\mathrm{L}_{\alpha}, \mathrm{TAP}, 120-269\right)$ - celestite, Si (K, TAP, 82-95) orthoclase, $\mathrm{Al}\left(\mathrm{K}_{\alpha}\right.$, TAP, 52-60) - albite, As ( $\mathrm{L}_{\alpha}$, TAP, 133-257) - GaAs, $\mathrm{Mg}\left(\mathrm{K}_{\alpha}\right.$, TAP, 48-101) - diopside, Lu ( $\mathrm{L}_{\alpha}$, LIFH, 204-210) - $\mathrm{LuPO}_{4}$, Ho ( $\left.\mathrm{L}_{\beta}, \mathrm{LIFH}, 355-365\right)-$ $\mathrm{HoPO}_{4}, \mathrm{Yb}\left(\mathrm{L}_{\alpha}, \mathrm{LIFH}, 186-189\right)-\mathrm{YbPO}_{4}, \mathrm{Tm}\left(\mathrm{L}_{\alpha}, \mathrm{LIFH}\right.$, 175-183) - $\mathrm{TmPO}_{4}, \mathrm{Er}\left(\mathrm{L}_{\alpha}, \mathrm{LIFH}, 173-176\right)-\mathrm{ErPO}_{4}, \mathrm{Gd}$ $\left(\mathrm{L}_{\beta}, \mathrm{LIFH}, 283-293\right)-\mathrm{GdPO}_{4}$, Dy ( $\left.\mathrm{L}_{\alpha}, \mathrm{LIFH}, 148-153\right)-$ $\mathrm{DyPO}_{4}, \mathrm{~Tb}\left(\mathrm{~L}_{\alpha}, \mathrm{LIFH}, 130-138\right)-\mathrm{TbPO}_{4}, \mathrm{Sm}\left(\mathrm{L}_{\beta}, \mathrm{LIFH}\right.$, 

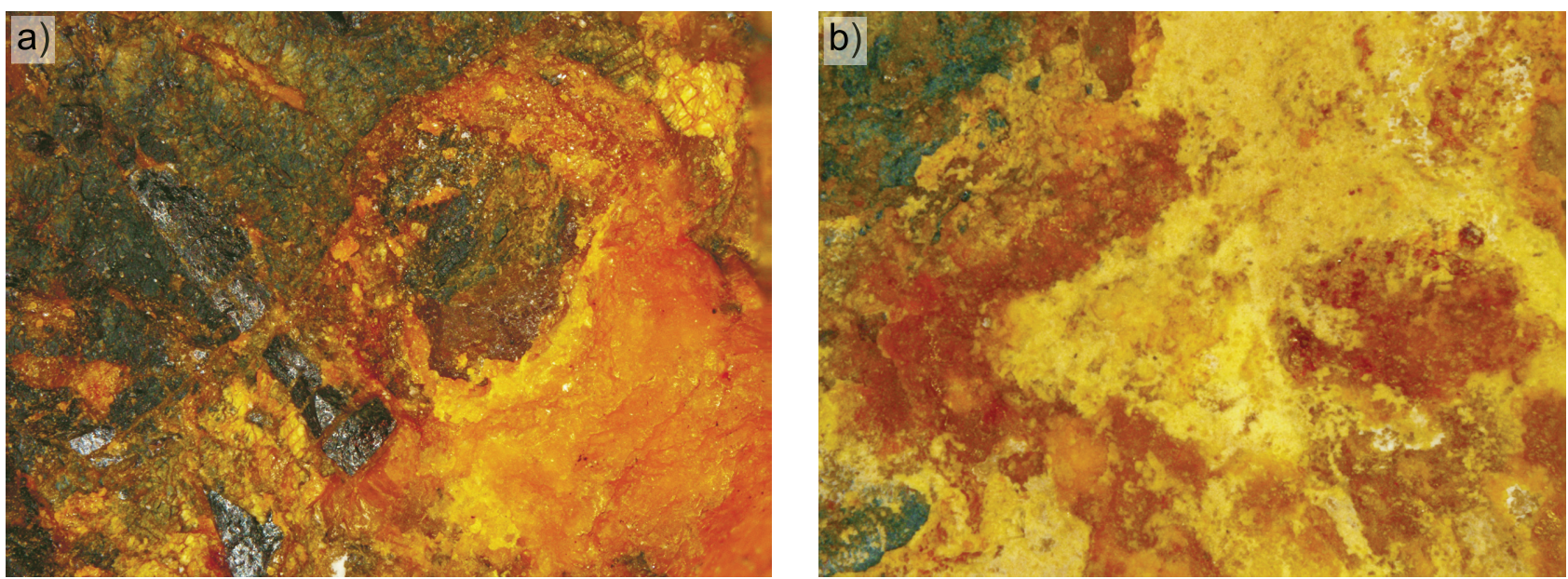

Fig. 2a - Brannerite (black) is replaced by the yellow-orange becquerelite. Horizontal width $4 \mathrm{~mm}$. b - Light yellow coatings of uranyl-oxide hydroxy-hydrates (dominance of vandendriesscheite and "calciolepersonnite") grown on the orange becquerelite crust. Black mineral is brannerite. Horizontal width $3 \mathrm{~mm}$.

251-269) - $\mathrm{SmPO}_{4}, \mathrm{Eu}\left(\mathrm{L}_{\alpha}, \mathrm{LIFH}, 130-135\right)-\mathrm{EuPO}_{4}, \mathrm{Pr}$ $\left(\mathrm{L}_{\beta}\right.$, LIFH, 230-242) - $\mathrm{PrPO}_{4}, \mathrm{Nd}\left(\mathrm{L}_{\alpha}, \mathrm{LIFH}, 123-127\right)-$ $\mathrm{NdPO}_{4}, \mathrm{Ce}\left(\mathrm{L}_{\alpha}, \mathrm{LIFH}, 121-130\right)-\mathrm{CePO}_{4}, \mathrm{La}\left(\mathrm{L}_{\alpha}, \mathrm{LIFH}\right.$, 130-139) - $\mathrm{LaPO}_{4}$, Co (K, LIFH, 94-109) - Co, Zn (K, LIF, 308-372) - gahnite, $\mathrm{Cu}\left(\mathrm{K}_{\alpha}\right.$, LIF, 214-253) - cuprite, Fe (K, LIF, 225-297) - olivine, Ti ( $\mathrm{K}_{a}$, LIF, 211-247) - rutile, Ba (L, LIF, 546-864) - baryte, Bi (M, , PETJ, $310-664)-\mathrm{Bi}_{2} \mathrm{Se}_{3}$.

$\mathrm{X}$-ray element distribution maps of uranyl-oxide hydroxy-hydrates were obtained (the same device) with an accelerating voltage of $15 \mathrm{kV}$ and probe current of $15 \mathrm{nA}$.

\section{Nature of uranyl minerals}

Uranyl minerals form conspicuously yellow coatings on the surface of the massive brannerite (+quartz) gangue, and fine crystalline crusts, with thickness up to $1 \mathrm{~mm}$.
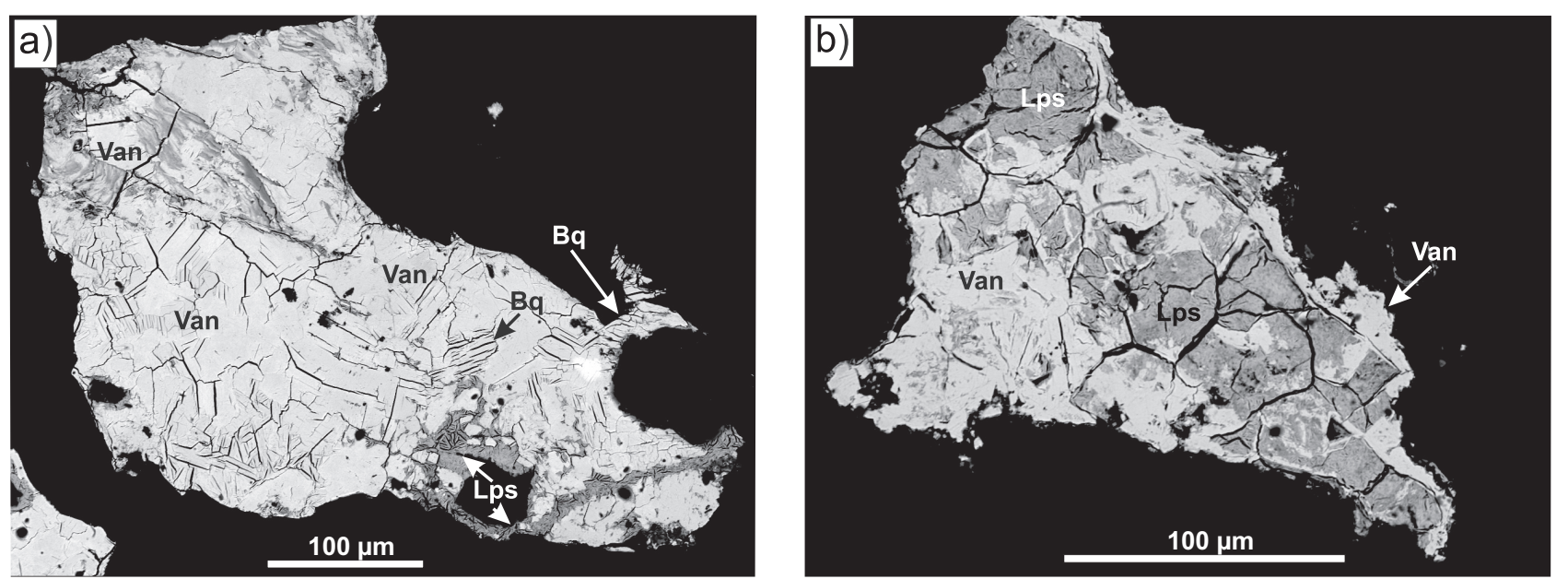

Fig. 3 Back-scattered electron images (BSE) of uranyl minerals from the Krátka Dolina Valley (Gemerská Poloma). a - Vandendriesscheite (Van) -

becquerelite (Bq) aggregate is cut by the "calciolepersonnite" veinlets (Lps). b - Intergrowth of "calciolepersonnite" (Lps) and vandendriesscheite (Van).

They also fill hairline fissures in brannerite. Using binocular magnifier it can be observed that the crust consists of two layers.

The lower one that covers directly brannerite surface is microcrystalline to compact, with yellow-orange colour and greasy lustre (Fig. 2a). It is formed by becquerelite far predominant over vandendriesscheite and leesite-like phase.

The upper layer is microcrystalline, with pale yellow colour and matte lustre (Fig. 2b). It is composed of dominant vandendriesscheite and insignificant amount of becquerelite. Uranyl-oxide hydroxy-hydrates in both layers are accompanied by lepersonnite-like mineral (an unnamed uranyl carbonate-silicate), although it is more widespread in the upper layer.

On a microscopic scale, becquerelite and vandendriesscheite form irregular aggregates that are difficult to distinguish in the BSE mode. Both minerals have a relatively 


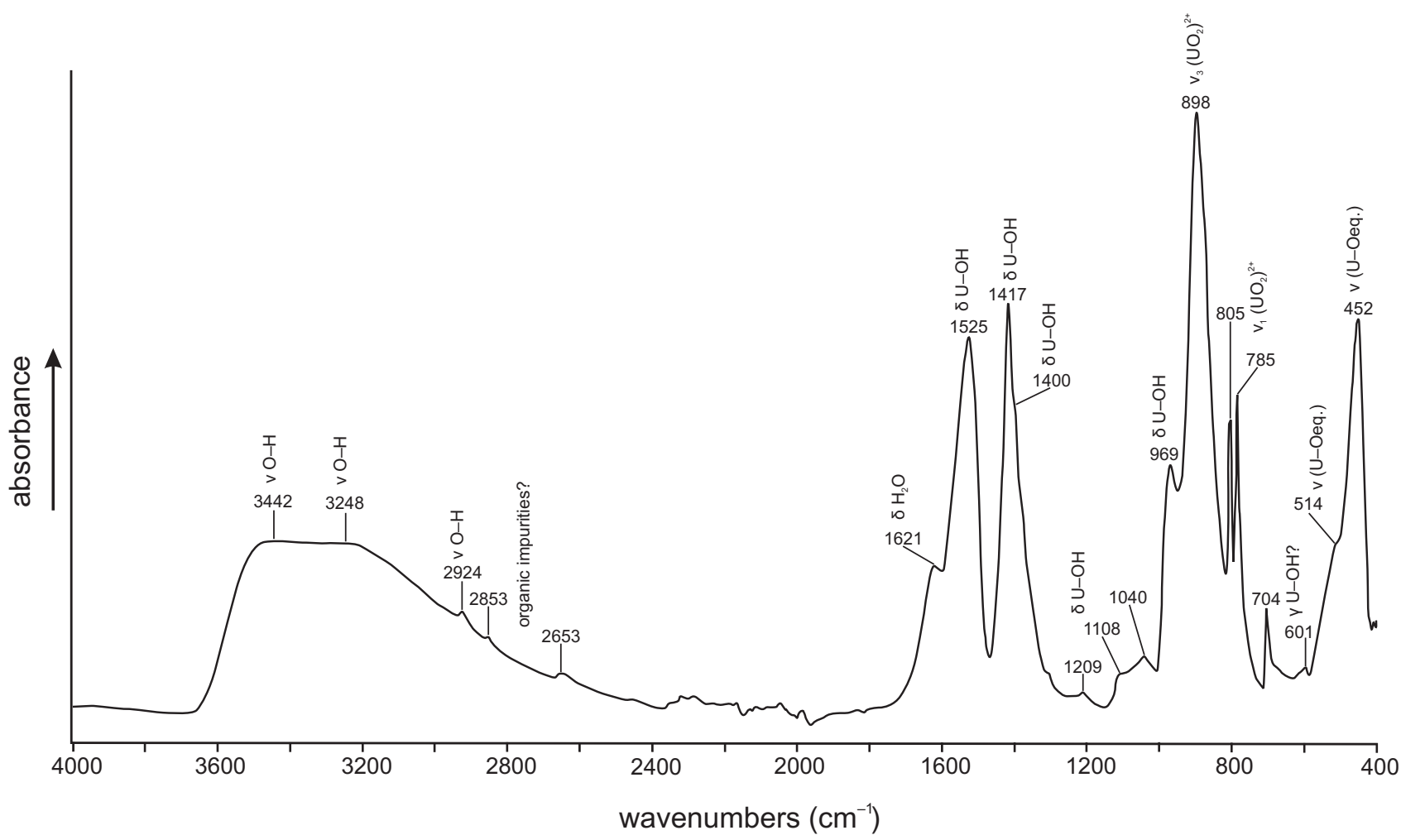

Fig. 4 Infrared spectrum of becquerelite from Gemerská Poloma.

Tab. 1 X-ray powder diffraction pattern of becquerelite from Gemerská Poloma

\begin{tabular}{rccccc}
\hline$I_{\text {obs. }}$ & $d_{\text {obs. }}$ & $d_{\text {calc. }}$ & $h$ & $k$ & $l$ \\
\hline 100.0 & 7.4683 & 7.4600 & 0 & 0 & 2 \\
1.0 & 6.5728 & 6.5689 & 1 & 0 & 2 \\
0.1 & 5.5972 & 5.5968 & 2 & 1 & 1 \\
0.9 & 4.6817 & 4.6811 & 1 & 0 & 3 \\
18.6 & 3.7324 & 3.7300 & 0 & 0 & 4 \\
1.6 & 3.6014 & 3.6018 & 1 & 0 & 4 \\
0.6 & 3.4582 & 3.4650 & 4 & 0 & 0 \\
0.3 & 3.3782 & 3.3752 & 4 & 0 & 1 \\
1.7 & 3.2019 & 3.1898 & 2 & 3 & 2 \\
0.5 & 3.1379 & 3.1426 & 4 & 0 & 2 \\
0.2 & 3.0974 & 3.0842 & 1 & 3 & 3 \\
0.1 & 2.9550 & 2.9589 & 4 & 2 & 1 \\
0.3 & 2.9173 & 2.9172 & 1 & 0 & 5 \\
0.4 & 2.5701 & 2.5806 & 4 & 2 & 3 \\
0.2 & 2.5377 & 2.5386 & 4 & 0 & 4 \\
1.2 & 2.4873 & 2.4867 & 0 & 0 & 6 \\
0.4 & 2.4480 & 2.4476 & 1 & 0 & 6 \\
0.1 & 2.3841 & 2.3769 & 1 & 3 & 5 \\
0.2 & 2.1082 & 2.1016 & 1 & 3 & 6 \\
0.4 & 2.0378 & 2.0372 & 2 & 0 & 7 \\
0.1 & 1.9356 & 1.9354 & 3 & 0 & 7 \\
0.5 & 1.8668 & 1.8656 & 6 & 3 & 3 \\
0.5 & 1.8504 & 1.8510 & 5 & 0 & 6 \\
0.2 & 1.6133 & 1.6123 & 2 & 0 & 9 \\
\hline & & & & &
\end{tabular}

compact surface; in cases, they can be recognized by cleavage or fissure systems (Fig. 3a-b). Becquerelite is characterized by either parallel cracks or fissures arranged with an approximate angle of $120^{\circ}$. The cracks in vandendriesscheite adopt a zig-zag arrangement and, unlike becquerelite, do not prefer a specific direction. Both minerals locally intergrow intimately.

Becquerelite was identified by the XRD analysis, IR spectroscopy, and also by the EPMA. The XRD pattern of becquerelite from the Krátka Dolina Valley (Tab. 1) is in line with previously published data (Frondel and Cuttitta 1953; Protas 1957; Christ and Clark 1960; Piret-Meunier and Piret 1982; Pagoaga et al. 1987; Burns and Li 2002). The unit-cell parameters of the studied becquerelite and their mutual ratios (Tab. 2) correspond to the published data for this mineral $(a: b: c=1.118: 1: 1.205$ - average calculated from $a, b, c$ values in Tab. 2).

In the IR vibration spectrum of studied becquerelite (Fig. 4) several bands were detected. Split band with peaks at 805 and $785 \mathrm{~cm}^{-1}$ can be connected with the $v_{1}$ $\left(\mathrm{UO}_{2}\right)^{2+}$ symmetric stretching vibration, the calculated $\mathrm{U}-\mathrm{O}$ bond lengths in uranyl ion are $1.81 \AA / 805 \mathrm{~cm}^{-1}$ and $1.83 \AA / 785 \mathrm{~cm}^{-1}$, respectively. A band with a high intensity at $898 \mathrm{~cm}^{-1}$ is related to the $v_{3}\left(\mathrm{UO}_{2}\right)^{2+}$ antisymmetric stretching vibrations; the corresponding $\mathrm{U}-\mathrm{O}$ bond length calculated from the observed stretching frequency is $1.79 \AA$. The obtained values of $\mathrm{U}-\mathrm{O}$ bond lengths in the studied becquerelite are in agreement with 
Tab. 2 Refined unit-cell parameters of becquerelite from Gemerská Poloma (orthorhombic space group $\operatorname{Pn} 2, a)$

\begin{tabular}{llllll}
\hline & \multicolumn{1}{c}{$a[\AA]$} & \multicolumn{1}{c}{$b[\AA]$} & \multicolumn{1}{c}{$c[\AA]$} & $V\left[\AA^{3}\right]$ & $a: b: c$ \\
\hline this work & $13.837(1)$ & $12.401(4)$ & $14.926(1)$ & $2561(1)$ & $1.116: 1: 1.204$ \\
Billiet and de Jong (1935) & 13.9 & 12.55 & 14.9 & 2600 & $1.108: 1: 1.187$ \\
Frondel and Cuttitta (1953) & $13.92(1)$ & $12.45(1)$ & $15.09(1)$ & 2615 & $1.118: 1: 1.212$ \\
Protas (1957) & $13.86(3)$ & $12.42(3)$ & $14.96(3)$ & 2575 & $1.116: 1: 1.205$ \\
Christ and Clark (1960) & 13.86 & 12.38 & 14.96 & 2567 & $1.120: 1: 1.208$ \\
Piret-Meunier and Piret (1982) & 13.86 & 12.3 & 14.92 & 2543.5 & $1.127: 1: 1.213$ \\
Pagoaga et al. (1987) & $13.8378(9)$ & $12.3781(12)$ & $14.9238(9)$ & $2556.2(1)$ & $1.118: 1: 1.206$ \\
Burns and Li (2002) & $13.8527(5)$ & $12.3929(4)$ & $14.9297(5)$ & $2563.2(1)$ & $1.118: 1: 1.205$ \\
\hline
\end{tabular}

published structure data (Pagoaga et al. 1987; Burns and Li 2002), or the results of the Raman spectroscopy study (Frost et al. 2007). Band at $452 \mathrm{~cm}^{-1}$ with a shoulder at $514 \mathrm{~cm}^{-1}$ may be assigned to the $v\left(\mathrm{U}-\mathrm{O}_{\text {equatorial }}\right)$ bending vibrations. Intense bands at 1525 and $1417 \mathrm{~cm}^{-1}$, with a shoulder at $1400 \mathrm{~cm}^{-1}$ were (sensu Frost et al. 2007) assigned to overtones or combination bands, but they may also be attributed to the $\delta \mathrm{U}-\mathrm{OH}$ bending vibrations. Weak bands at 1209, 1108, 1040, and $969 \mathrm{~cm}^{-1}$ may also be connected with $\delta \mathrm{U}-\mathrm{OH}$ bending vibrations. Bands at the 704 and $601 \mathrm{~cm}^{-1}$ probably represent the $\gamma \mathrm{U}-\mathrm{OH}$ out-of-plane bending vibration or the water molecules librations. The $\delta \mathrm{H}_{2} \mathrm{O}$ bending vibration is manifested by the band at $1621 \mathrm{~cm}^{-1}$, bands at 3442, 3248 and $2924 \mathrm{~cm}^{-1}$ may be assigned to the $v$ O-H stretching vibrations. Wavenumbers of the $v \mathrm{O}-\mathrm{H}$ vibrations were used for calculation of $\mathrm{O}-\mathrm{H} \cdots \mathrm{O}$ hydrogen bond lengths (after Libowitzky 1999). The calculated hydrogen bond lengths $d(\mathrm{O} \cdots \mathrm{O}) 2.83 \AA / 3442 \mathrm{~cm}^{-1} ; 2.72 \AA / 3248 \mathrm{~cm}^{-1}$ and $2.63 \AA / 2924 \mathrm{~cm}^{-1}$ correspond to rather strong hydrogen bonds. Weak bands at 2853 and $2653 \mathrm{~cm}^{-1}$ may be assigned to organic impurities in a sample. Overall, the spectrum is in a good agreement with published IR spectra of becquerelite (Čejka et al. 1998; Frost et al. 2007).

Based on their chemical composition, two types of becquerelite (I and II) can be distinguished in the samples studied. Becquerelite I (Tab. 3 , analyses 1 to 6 ) is characterized by high $\mathrm{CaO}$ (average of 2.44 wt. \%; 0.85 apfu $\mathrm{Ca})$, and its composition is close to the ideal becquerelite formula (Fig. 5a-b); the total content of other divalent cations reaches 0.42 wt. $\% \mathrm{M}^{2+} \mathrm{O}$ (corresponding to 0.06 apfu $\mathrm{M}^{2+}$ ). Only insignificant amount of $\mathrm{K}_{2} \mathrm{O}$ (average 0.18 wt. \%; 0.04 apfu $\mathrm{K}$ ) is present at the cationic (A) position,. The average chemical composition of becquerelite I can be expressed by the empirical formula: $\left(\mathrm{Ca}_{0.85} \mathrm{~K}_{0.04} \mathrm{Na}_{0.01} \mathrm{Fe}_{0.02} \mathrm{Zn}_{0.02} \mathrm{Ba}_{0.01}\right.$ $\left.\mathrm{Pb}_{0.01}\right)_{\Sigma 0.96}\left[\left(\mathrm{UO}_{2}\right)_{6} \mathrm{O}_{4}(\mathrm{OH})_{5.84}\right] \cdot 8 \mathrm{H}_{2} \mathrm{O}$.

Becquerelite II is characterized by increased $\mathrm{K}_{2} \mathrm{O}$ (average of 1.29 wt. \%; 0.27 apfu K; Tab. 3, analyses 7-9) at the expense of $\mathrm{CaO}$ (c. 1.01 wt. \%; 0.36 apfu Ca). Compared with becquerelite I, the $M^{2+}$ content (excluding Ca) is slightly increased (average 0.40 wt. $\% \mathrm{M}^{2+} \mathrm{O} ; 0.14 \mathrm{apfu}$ $M^{2+}$ ). An elevated content of $\mathrm{Bi}\left(0.51\right.$ wt. $\% \mathrm{Bi}_{2} \mathrm{O}_{3} ; 0.02$ apfu $\mathrm{Bi}$ ) was found sporadically. Becquerelite II contains a higher proportion of compreignacite molecule in the structure (Fig. 5a-b); the average chemical formula can be expressed as: $\left(\mathrm{Ca}_{0.36} \mathrm{~K}_{0.27} \mathrm{Na}_{0.01} \mathrm{Fe}_{0.02} \mathrm{Zn}_{0.02} \mathrm{~Pb}_{0.01} \mathrm{Bi}_{0.01}\right)_{\Sigma 0.78}$ $\left[\left(\mathrm{UO}_{2}\right)_{6} \mathrm{O}_{4}(\mathrm{OH})_{5.29}\right] \cdot 8 \mathrm{H}_{2} \mathrm{O}$. The $\mathrm{Si}, \mathrm{P}$ and As contents in a)

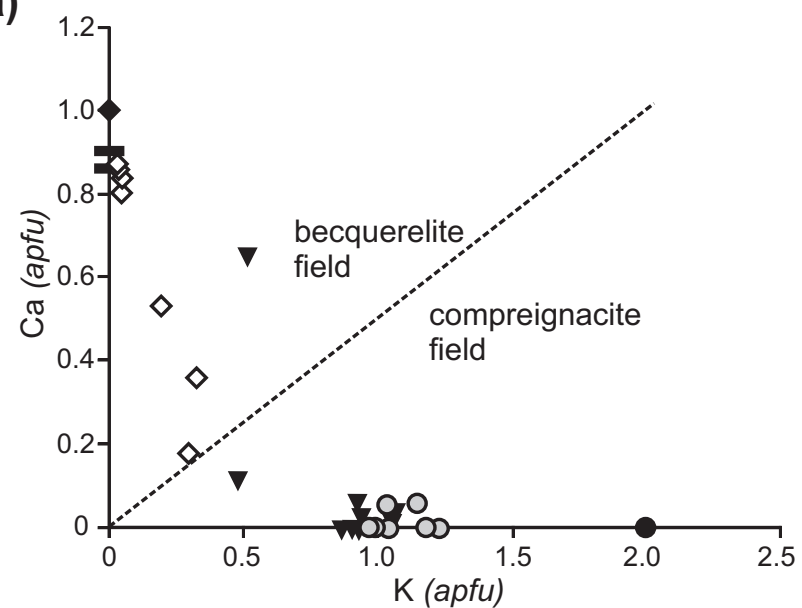

b)

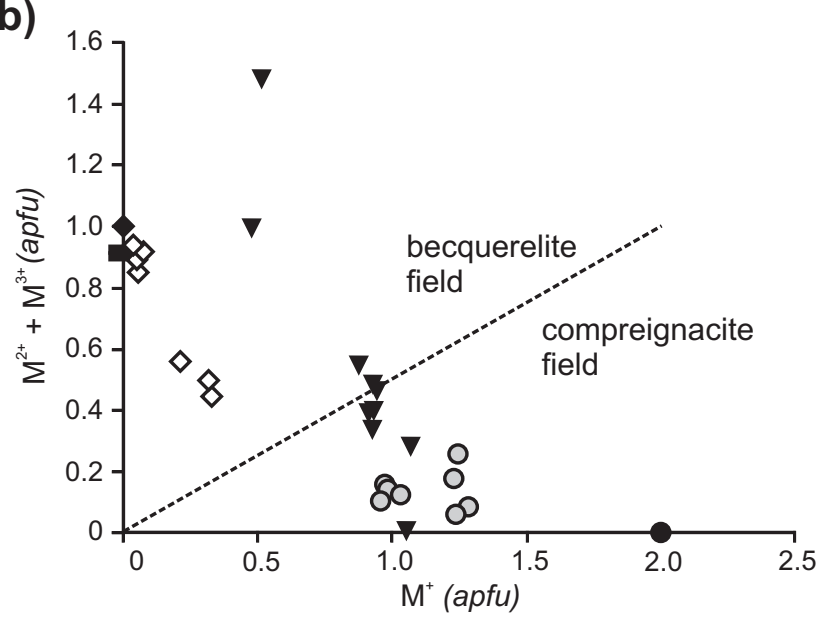

$\diamond$ becquerelite (this work)

becquerelite (ideal. comp.) compreignacite (ideal. comp.)

becquerelite (Finch 1994) o compreignacite (Sejkora et al. 2013)

v compreignacite (Plášil et al. 2014)

Fig. 5 Becquerelite compositions $(a p f u)$ from Gemerská Poloma compared to previously published data for becquerelite and compreignacite. a - K vs. Ca diagram. $\mathbf{b}-M^{+}$vs. $M^{2+}+M^{3+}$ diagram. 
both types of becquerelite are negligible (below the respective detection limits).

Vandendriesscheite-like mineral was determined only by the microprobe analysis (Tab. 4). Vandendriesscheite can be expressed by the ideal formula $\mathrm{Pb}_{1.5}\left(\mathrm{UO}_{2}\right)_{10} \mathrm{O}_{6}(\mathrm{OH})_{11} \cdot 11 \mathrm{H}_{2} \mathrm{O}$ (Vaes 1947; Burns 1997). In the samples studied, the dominant element in the cationic (A) position is $\mathrm{Pb}$ in variable concentrations (6.02-8.49 wt. \% $\mathrm{PbO}$; 0.93-1.32 apfu $\mathrm{Pb}$ ). Lead shows a significant negative correlation $(r=-0.78)$ with the second most abundant element - potassium (Fig. 6a) (1.13-1.57 wt. \% $\mathrm{K}_{2} \mathrm{O}$; 0.43-0.58 apfu K). Contents of $\mathrm{BaO}, \mathrm{FeO}, \mathrm{Al}_{2} \mathrm{O}_{3}$, and $\mathrm{SiO}_{2}$ do not reach higher concentrations $(<0.7$ wt. $\%$ for each oxide; Tab. 4). These do not have a significant effect on the overall chemical composition of vandendriesscheite (Fig. 6b). The average chemical composition of the studied vandendriesscheite can be expressed as follows: $\left(\mathrm{K}_{0.49} \mathrm{Na}_{0.02}\right)_{\Sigma 0.51}$ $\left(\mathrm{Pb}_{1.20} \mathrm{Fe}_{0.05} \mathrm{Zn}_{0.04} \mathrm{~B} \mathrm{a}_{0.03}\right.$ $\left.\mathrm{Sr}_{0.02} \mathrm{Al}_{0.02}\right)_{\Sigma 1.36}\left[\left(\mathrm{UO}_{2}\right)_{10} \mathrm{O}_{6}\right.$ $\left.\left(\mathrm{SiO}_{4}\right)_{0.05}\left(\mathrm{PO}_{4}\right)_{0.02}(\mathrm{OH})_{10.86}\right]$. $11 \mathrm{H}_{2} \mathrm{O}$.

Tab. 3 Chemical composition of becquerelite from Gemerská Poloma (empirical formulae calculated on the basis of $6 \mathrm{U}$ apfu

\begin{tabular}{|c|c|c|c|c|c|c|c|c|c|c|c|}
\hline & 1 & 2 & 3 & 4 & 5 & 6 & Mean & 7 & 8 & 9 & Mean \\
\hline $\mathrm{Na}_{2} \mathrm{O}$ & 0.02 & 0.00 & 0.00 & 0.02 & 0.10 & 0.03 & 0.03 & 0.05 & 0.05 & 0.01 & 0.04 \\
\hline $\mathrm{K}_{2} \mathrm{O}$ & 0.17 & 0.15 & 0.22 & 0.20 & 0.19 & 0.14 & 0.18 & 0.95 & 1.39 & 1.52 & 1.29 \\
\hline $\mathrm{CaO}$ & 2.45 & 2.57 & 2.43 & 2.18 & 2.46 & 2.57 & 2.44 & 1.54 & 0.50 & 1.00 & 1.01 \\
\hline $\mathrm{SrO}$ & 0.00 & 0.02 & 0.00 & 0.00 & 0.00 & 0.00 & 0.00 & 0.00 & 0.02 & 0.00 & 0.01 \\
\hline $\mathrm{BaO}$ & 0.21 & 0.00 & 0.00 & 0.14 & 0.21 & 0.00 & 0.09 & 0.00 & 0.00 & 0.00 & 0.00 \\
\hline $\mathrm{FeO}$ & 0.08 & 0.15 & 0.14 & 0.00 & 0.05 & 0.10 & 0.09 & 0.00 & 0.23 & 0.07 & 0.10 \\
\hline $\mathrm{ZnO}$ & 0.00 & 0.11 & 0.09 & 0.05 & 0.00 & 0.17 & 0.07 & 0.09 & 0.04 & 0.09 & 0.08 \\
\hline $\mathrm{PbO}$ & 0.00 & 0.00 & 0.00 & 0.23 & 0.14 & 0.00 & 0.06 & 0.06 & 2.68 & 0.23 & 0.99 \\
\hline $\mathrm{Bi}_{2} \mathrm{O}_{3}$ & 0.00 & 0.00 & 0.00 & 0.09 & 0.00 & 0.00 & 0.02 & 0.00 & 0.00 & 0.51 & 0.17 \\
\hline $\mathrm{SiO}_{2}$ & 0.00 & 0.00 & 0.00 & 0.00 & 0.07 & 0.00 & 0.01 & 0.00 & 0.00 & 0.00 & 0.00 \\
\hline $\mathrm{P}_{2} \mathrm{O}_{5}$ & 0.00 & 0.01 & 0.05 & 0.03 & 0.02 & 0.03 & 0.02 & 0.00 & 0.00 & 0.00 & 0.00 \\
\hline $\mathrm{As}_{2} \mathrm{O}_{5}$ & 0.00 & 0.00 & 0.03 & 0.01 & 0.00 & 0.00 & 0.01 & 0.06 & 0.02 & 0.00 & 0.03 \\
\hline $\mathrm{UO}_{3}$ & 87.89 & 90.23 & 89.21 & 83.61 & 86.96 & 90.43 & 88.05 & 88.83 & 85.40 & 85.53 & 86.59 \\
\hline $\mathrm{H}_{2} \mathrm{O}^{*}$ & 8.14 & 8.13 & 8.14 & 8.13 & 8.11 & 8.13 & 8.13 & 8.19 & 7.00 & 8.17 & 7.79 \\
\hline$\Sigma$ wt. $\%$ & 98.96 & 101.37 & 100.33 & 94.68 & 98.29 & 101.60 & 99.19 & 99.77 & 97.33 & 97.14 & 98.08 \\
\hline $\mathrm{Na}$ & 0.005 & 0.000 & 0.000 & 0.007 & 0.031 & 0.009 & 0.009 & 0.015 & 0.017 & 0.003 & 0.012 \\
\hline K & 0.036 & 0.030 & 0.045 & 0.043 & 0.039 & 0.027 & 0.037 & 0.195 & 0.297 & 0.324 & 0.272 \\
\hline $\mathrm{Ca}$ & 0.851 & 0.872 & 0.835 & 0.799 & 0.864 & 0.871 & 0.849 & 0.531 & 0.178 & 0.359 & 0.356 \\
\hline $\mathrm{Sr}$ & 0.000 & 0.004 & 0.000 & 0.000 & 0.000 & 0.000 & 0.001 & 0.000 & 0.005 & 0.000 & 0.002 \\
\hline $\mathrm{Ba}$ & 0.027 & 0.000 & 0.000 & 0.019 & 0.026 & 0.000 & 0.012 & 0.000 & 0.000 & 0.000 & 0.000 \\
\hline $\mathrm{Fe}$ & 0.023 & 0.039 & 0.038 & 0.000 & 0.013 & 0.027 & 0.023 & 0.000 & 0.065 & 0.020 & 0.028 \\
\hline $\mathrm{Zn}$ & 0.000 & 0.027 & 0.022 & 0.011 & 0.000 & 0.040 & 0.018 & 0.022 & 0.011 & 0.023 & 0.019 \\
\hline $\mathrm{Pb}$ & 0.000 & 0.000 & 0.000 & 0.021 & 0.012 & 0.000 & 0.005 & 0.005 & 0.241 & 0.021 & 0.089 \\
\hline $\mathrm{Bi}$ & 0.000 & 0.000 & 0.000 & 0.004 & 0.000 & 0.000 & 0.000 & 0.000 & 0.000 & 0.022 & 0.007 \\
\hline$\sum$ A site & 0.942 & 0.971 & 0.940 & 0.905 & 0.986 & 0.975 & 0.953 & 0.768 & 0.814 & 0.772 & 0.784 \\
\hline $\mathrm{SiO}_{4}^{4-}$ & 0.000 & 0.000 & 0.000 & 0.000 & 0.024 & 0.000 & 0.004 & 0.000 & 0.000 & 0.000 & 0.000 \\
\hline $\mathrm{PO}_{4}^{3-}$ & 0.001 & 0.002 & 0.007 & 0.004 & 0.003 & 0.004 & 0.003 & 0.000 & 0.000 & 0.000 & 0.000 \\
\hline $\mathrm{AsO}_{4}^{3-}$ & 0.000 & 0.000 & 0.003 & 0.001 & 0.000 & 0.000 & 0.001 & 0.005 & 0.001 & 0.000 & 0.002 \\
\hline$\Sigma \mathrm{T}$ site & 0.001 & 0.002 & 0.010 & 0.005 & 0.027 & 0.004 & 0.008 & 0.005 & 0.001 & 0.000 & 0.002 \\
\hline $\mathrm{UO}_{2}^{2+}$ & 6.000 & 6.000 & 6.000 & 6.000 & 6.000 & 6.000 & 6.000 & 6.000 & 6.000 & 6.000 & 6.000 \\
\hline $\mathrm{OH}^{* *}$ & 5.841 & 5.908 & 5.805 & 5.751 & 5.809 & 5.901 & 5.836 & 5.310 & 5.309 & 5.239 & $\overline{5.286}$ \\
\hline $\mathrm{H}_{2} \mathrm{O}$ & 8.000 & 8.000 & 8.000 & 8.000 & 8.000 & 8.000 & 8.000 & 8.000 & 8.000 & 8.000 & 8.000 \\
\hline
\end{tabular}

a)

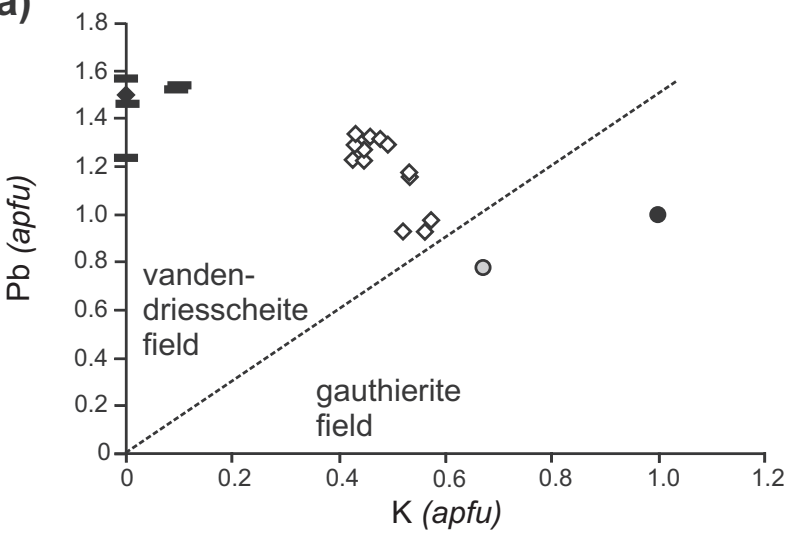

b)

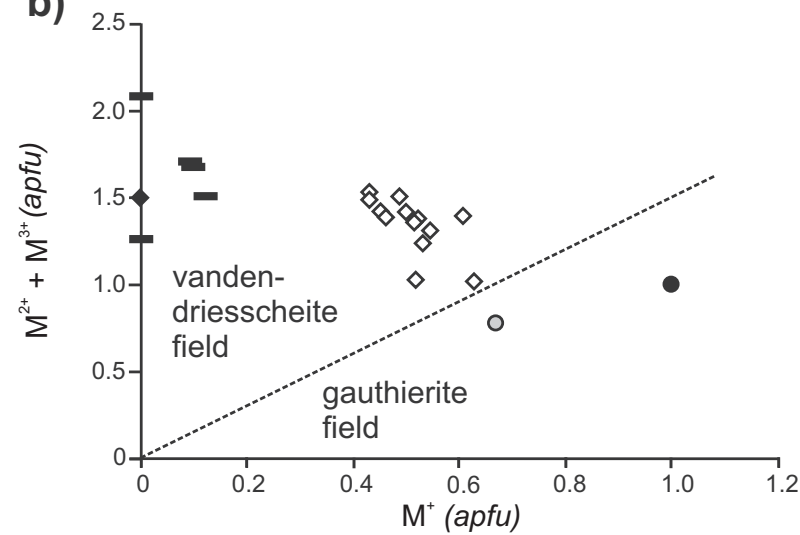

$\diamond \quad$ vandendriesscheite (this work)

gauthierite (ideal. comp., Olds et al. 2017a)

- vandendriesscheite (Finch 1994)

vandendriesscheite (ideal. comp.)

- gauthierite (Olds et al. 2017a)

Fig. 6 Vandendriesscheite compositions (apfu) from the Gemerská Poloma compared with published data for vandendriesscheite and gauthierite. a $-\mathrm{K}$ vs. Pb diagram. b- $M^{+}$vs. $M^{2+}+M^{\beta+}$ diagram. 
Tab. 4 Chemical composition of vandendriesscheite from Gemerská Poloma (empirical formulae calculated on the basis of 10 U apfu)

\begin{tabular}{|c|c|c|c|c|c|c|c|c|c|c|c|c|c|c|c|}
\hline & 1 & 2 & 3 & 4 & 5 & 6 & 7 & 8 & 9 & 10 & 11 & 12 & 13 & 14 & Mean \\
\hline $\mathrm{Na}_{2} \mathrm{O}$ & 0.02 & 0.00 & 0.02 & 0.00 & 0.00 & 0.14 & 0.00 & 0.12 & 0.00 & 0.02 & 0.02 & 0.06 & 0.00 & 0.08 & 0.03 \\
\hline $\mathrm{K}_{2} \mathrm{O}$ & 1.45 & 1.14 & 1.18 & 1.25 & 1.16 & 1.20 & 1.13 & 1.55 & 1.43 & 1.35 & 1.31 & 1.57 & 1.48 & 1.30 & 1.32 \\
\hline $\mathrm{CaO}$ & 0.00 & 0.00 & 0.00 & 0.00 & 0.00 & 0.00 & 0.00 & 0.00 & 0.01 & 0.00 & 0.00 & 0.00 & 0.00 & 0.00 & 0.00 \\
\hline $\mathrm{SrO}$ & 0.15 & 0.06 & 0.00 & 0.03 & 0.06 & 0.09 & 0.00 & 0.00 & 0.07 & 0.04 & 0.06 & 0.03 & 0.01 & 0.00 & 0.04 \\
\hline $\mathrm{BaO}$ & 0.00 & 0.31 & 0.00 & 0.00 & 0.08 & 0.18 & 0.55 & 0.00 & 0.00 & 0.00 & 0.22 & 0.36 & 0.00 & 0.00 & 0.12 \\
\hline $\mathrm{FeO}$ & 0.14 & 0.11 & 0.15 & 0.06 & 0.08 & 0.00 & 0.12 & 0.08 & 0.04 & 0.09 & 0.16 & 0.33 & 0.05 & 0.10 & 0.11 \\
\hline $\mathrm{ZnO}$ & 0.00 & 0.21 & 0.10 & 0.06 & 0.10 & 0.04 & 0.07 & 0.12 & 0.11 & 0.14 & 0.13 & 0.09 & 0.11 & 0.01 & 0.09 \\
\hline $\mathrm{PbO}$ & 7.58 & 7.73 & 7.76 & 8.51 & 8.46 & 8.11 & 8.06 & 6.03 & 6.02 & 8.45 & 8.48 & 6.32 & 7.64 & 8.49 & 7.69 \\
\hline $\mathrm{Bi}_{2} \mathrm{O}_{3}$ & 0.00 & 0.00 & 0.00 & 0.00 & 0.00 & 0.05 & 0.00 & 0.00 & 0.00 & 0.00 & 0.00 & 0.00 & 0.00 & 0.00 & 0.00 \\
\hline $\mathrm{Al}_{2} \mathrm{O}_{3}$ & 0.03 & 0.12 & 0.19 & 0.00 & 0.11 & 0.00 & 0.00 & 0.00 & 0.00 & 0.00 & 0.00 & 0.36 & 0.00 & 0.00 & 0.06 \\
\hline $\mathrm{SiO}_{2}$ & 0.00 & 0.26 & 0.18 & 0.00 & 0.20 & 0.00 & 0.00 & 0.00 & 0.00 & 0.00 & 0.00 & 0.63 & 0.00 & 0.04 & 0.09 \\
\hline $\mathrm{P}_{2} \mathrm{O}_{5}$ & 0.00 & 0.10 & 0.00 & 0.02 & 0.00 & 0.02 & 0.02 & 0.00 & 0.06 & 0.03 & 0.02 & 0.53 & 0.29 & 0.12 & 0.09 \\
\hline $\mathrm{As}_{2} \mathrm{O}_{5}$ & 0.00 & 0.02 & 0.00 & 0.00 & 0.00 & 0.00 & 0.02 & 0.09 & 0.00 & 0.00 & 0.00 & 0.13 & 0.00 & 0.14 & 0.03 \\
\hline $\mathrm{H}_{2} \mathrm{O}^{*}$ & 6.27 & 6.21 & 6.24 & 6.22 & 6.20 & 6.23 & 6.20 & 6.38 & 6.39 & 6.23 & 6.20 & 6.24 & 6.28 & 6.22 & 6.25 \\
\hline$\Sigma$ wt. $\%$ & 98.28 & 97.04 & 96.53 & 98.38 & 97.78 & 98.05 & 95.83 & 97.80 & 97.32 & 99.95 & 99.88 & 99.62 & 100.37 & 99.09 & 98.28 \\
\hline $\mathrm{Na}$ & 0.013 & 0.000 & 0.009 & 0.000 & 0.000 & 0.077 & 0.000 & 0.067 & 0.000 & 0.010 & 0.011 & 0.032 & 0.000 & 0.045 & 0.019 \\
\hline $\mathrm{K}$ & 0.533 & 0.430 & 0.444 & 0.461 & 0.433 & 0.445 & 0.430 & 0.563 & 0.520 & 0.491 & 0.478 & 0.575 & 0.532 & 0.479 & 0.487 \\
\hline $\mathrm{Ca}$ & 0.000 & 0.000 & 0.000 & 0.000 & 0.000 & 0.000 & 0.000 & 0.000 & 0.006 & 0.000 & 0.000 & 0.000 & 0.000 & 0.000 & 0.000 \\
\hline $\mathrm{Sr}$ & 0.051 & 0.020 & 0.000 & 0.010 & 0.020 & 0.029 & 0.000 & 0.000 & 0.025 & 0.014 & 0.021 & 0.010 & 0.004 & 0.000 & 0.015 \\
\hline $\mathrm{Ba}$ & 0.000 & 0.071 & 0.000 & 0.000 & 0.018 & 0.041 & 0.129 & 0.000 & 0.000 & 0.000 & 0.048 & 0.082 & 0.000 & 0.000 & 0.028 \\
\hline $\mathrm{Fe}$ & 0.067 & 0.052 & 0.073 & 0.029 & 0.037 & 0.000 & 0.059 & 0.036 & 0.020 & 0.045 & 0.076 & 0.159 & 0.023 & 0.049 & 0.052 \\
\hline $\mathrm{Zn}$ & 0.000 & 0.090 & 0.045 & 0.026 & 0.044 & 0.017 & 0.033 & 0.048 & 0.045 & 0.059 & 0.055 & 0.039 & 0.044 & 0.006 & 0.039 \\
\hline $\mathrm{Pb}$ & 1.176 & 1.226 & 1.231 & 1.327 & 1.333 & 1.267 & 1.297 & 0.926 & 0.928 & 1.295 & 1.306 & 0.977 & 1.158 & 1.317 & 1.197 \\
\hline $\mathrm{Bi}$ & 0.000 & 0.000 & 0.000 & 0.000 & 0.000 & 0.004 & 0.000 & 0.000 & 0.000 & 0.000 & 0.000 & 0.000 & 0.000 & 0.000 & 0.000 \\
\hline $\mathrm{Al}$ & 0.008 & 0.042 & 0.066 & 0.000 & 0.039 & 0.000 & 0.000 & 0.000 & 0.000 & 0.000 & 0.000 & 0.120 & 0.000 & 0.000 & 0.020 \\
\hline$\Sigma$ A site & 1.850 & 1.931 & 1.868 & 1.852 & 1.924 & 1.880 & 1.947 & 1.640 & 1.543 & 1.914 & 1.994 & 1.995 & 1.762 & 1.895 & 1.857 \\
\hline $\mathrm{PO}_{4}^{3-}$ & 0.000 & 0.024 & 0.001 & 0.006 & 0.000 & 0.004 & 0.006 & 0.000 & 0.015 & 0.007 & 0.005 & 0.130 & 0.068 & 0.029 & 0.021 \\
\hline $\mathrm{AsO}_{4}^{3-}$ & 0.000 & 0.002 & 0.000 & 0.000 & 0.000 & 0.000 & 0.004 & 0.014 & 0.000 & 0.000 & 0.000 & 0.020 & 0.000 & 0.021 & 0.004 \\
\hline$\Sigma \mathrm{T}$ site & 0.000 & 0.177 & 0.108 & 0.006 & 0.116 & 0.004 & 0.010 & 0.014 & 0.015 & 0.007 & 0.005 & 0.511 & 0.068 & 0.073 & 0.080 \\
\hline $\mathrm{UO}_{2}{ }^{2+}$ & 10.000 & 10.000 & 10.000 & 10.000 & 10.000 & 10.000 & 10.000 & 10.000 & 10.000 & 10.000 & 10.000 & 10.000 & 10.000 & 10.000 & 10.000 \\
\hline $\mathrm{OH}^{* *}$ & 11.161 & 10.791 & 10.917 & 11.226 & 10.990 & 11.229 & 11.435 & 10.608 & 10.522 & 11.306 & 11.484 & 9.611 & 10.788 & 10.023 & 10.864 \\
\hline $\mathrm{H}_{2} \mathrm{O}$ & 11.000 & 11.000 & 11.000 & 11.000 & 11.000 & 11.000 & 11.000 & 11.000 & 11.000 & 11.000 & 11.000 & 11.000 & 11.000 & 11.000 & 11.000 \\
\hline
\end{tabular}

$\mathrm{H}_{2} \mathrm{O}^{*}$ - calculation based on the theoretical content of $11 \mathrm{H}_{2} \mathrm{O}$ in ideal vandendriesscheite formula

$\mathrm{OH}^{* *}$ - calculation based on the charge-balance

The mineral phase chemically close to leesite (Olds et al. 2018) was found only rarely. A leesite-like phase forms irregular grains, not exceeding $50 \mu \mathrm{m}$ in size, enclosed by becquerelite and vandendriesscheite (see BSE image in Fig. 7). At the cationic (A) position, potassium is prevailing (2.58 wt. $\% \mathrm{~K}_{2} \mathrm{O} ; 0.72 a p f u \mathrm{~K}$; Tab. 5); the contents of $\mathrm{BaO}, \mathrm{FeO}, \mathrm{ZnO}$ and $\mathrm{PbO}$ are elevated only a little $(0.0 \mathrm{X}-1$ wt. $\%$, for each oxide). The empirical formula of leesite-like mineral can be expressed as: $\left(\mathrm{K}_{0.72}\right.$ $\left.\mathrm{Sr}_{0.01} \mathrm{Ba}_{0.02} \mathrm{Fe}_{0.03} \mathrm{Zn}_{0.01} \mathrm{~Pb}_{0.02} \mathrm{Al}_{0.02}\right)_{\Sigma 0.83}\left(\mathrm{H}_{2} \mathrm{O}\right)_{2}\left[\left(\mathrm{UO}_{2}\right)_{4} \mathrm{O}_{2}\right.$ $\left.\left(\mathrm{SiO}_{4}\right)_{0.01}(\mathrm{OH})_{5,00}\right]_{\Sigma 11,01} \cdot 3 \mathrm{H}_{2} \mathrm{O}$. The near-absence of $\mathrm{Ca}$ and $\mathrm{Pb}$ in leesite-like phase (Fig. 7) documents that this is not a "mixed" analysis of becquerelite and vandendriesscheite fine-intergrowths.

Unnamed mineral phase chemically close to lepersonnite- $(G d)$ was detected by EMPA. It is younger than the other uranyl-oxides hydroxy-hydrates, in which it forms veinlets or irregular nests and often replaces them along the fissures (Fig. 3a-b). Lepersonnite-like mineral aggregates, up to $250 \mu \mathrm{m}$ across, consist of needle-like crystals (up to $15 \mu \mathrm{m}$ ) arranged in fan-shape formations (Fig. 8a-b). The chemical composition of lepersonnite-like phase is given in Tab. 6. The Y + REE content ranges from 2.67 to 4.33 wt. $\% \mathrm{Y}_{2} \mathrm{O}_{3}$ and $\mathrm{REE}_{2} \mathrm{O}_{3}$ (0.69-1.11 apfu Y + REE). Yttrium is low (max. 0.08 wt. $\% \mathrm{Y}_{2} \mathrm{O}_{3} ; 0.03$ apfu $\mathrm{Y}$ ). Among REE, the dominant are $\mathrm{Sm}\left(1.07-1.55\right.$ wt. $\% \mathrm{Sm}_{2} \mathrm{O}_{3} ; 0.28-0.40$ apfu $\left.\mathrm{Sm}\right)$ and $\mathrm{Nd}\left(0.71-1.30\right.$ wt. $\% \mathrm{Nd}_{2} \mathrm{O}_{3} ; 0.19-0.36$ apfu $\left.\mathrm{Nd}\right)$. The average content of $\mathrm{Gd}_{2} \mathrm{O}_{3}$ is only 0.46 wt. \% (0.11 apfu $\mathrm{Gd}$ ). The concentrations each of the remaining REE ( $\mathrm{La}, \mathrm{Ce}, \mathrm{Pr}, \mathrm{Eu}, \mathrm{Tb}, \mathrm{Dy}, \mathrm{Tm}, \mathrm{Yb}$ ) do not exceed 0.36 wt. \% (for each element oxide). In the mineral structure, LREE (average 3.05 wt. \% LREE $_{2} \mathrm{O}_{3} ; 0.80$ apfu LREE) dominate over HREE (average 0.42 wt. $\% \mathrm{HREE}_{2} \mathrm{O}_{3}$; 

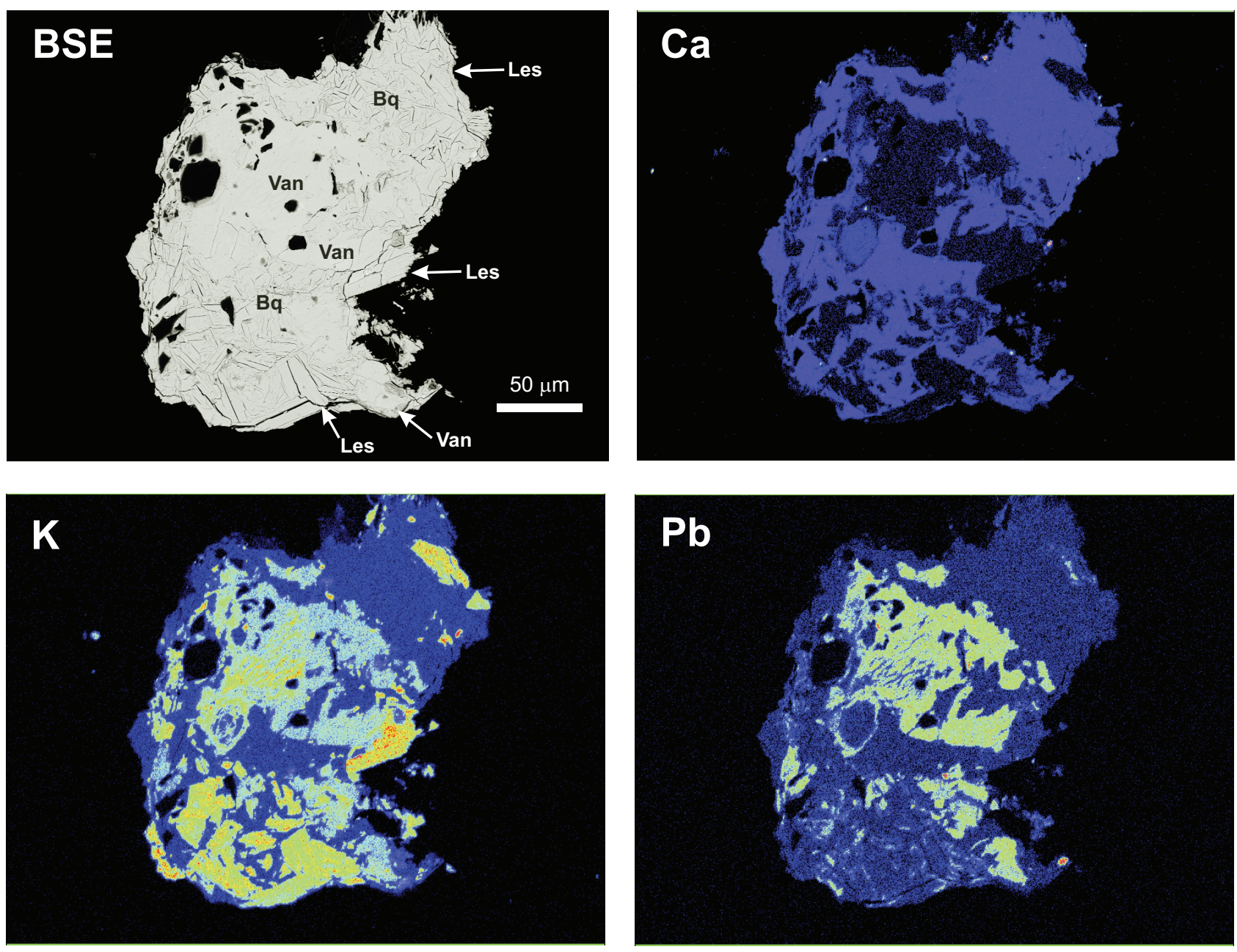

\section{element content increase}

Fig. 7 Back-scattered electron (BSE) images and $\mathrm{Ca}, \mathrm{K}$ and $\mathrm{Pb}$ X-ray element distribution maps of uranyl-oxide hydroxy-hydrates from Gemerská Poloma. $\mathrm{Bq}$ - becquerelite, Les - leesite-like phase, Van - vandendriesscheite.
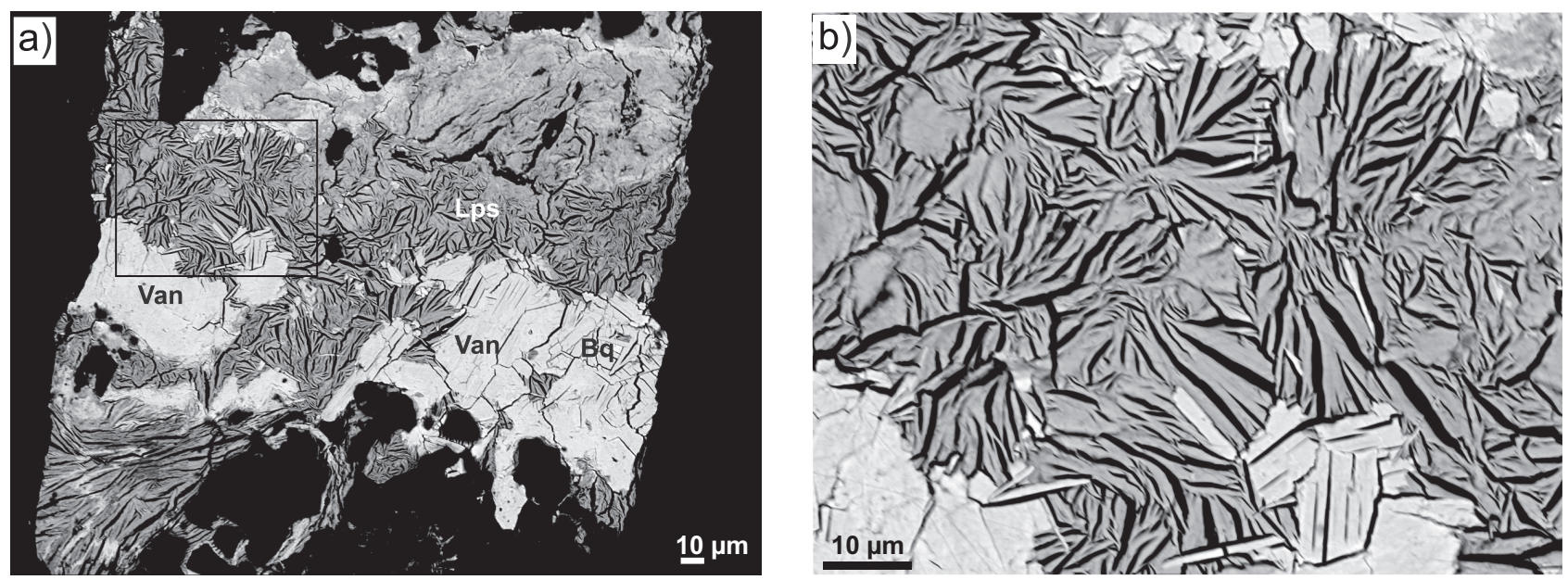

Fig. 8 Back-scattered electron (BSE) images of uranyl minerals from the Krátka Dolina Valley (Gemerská Poloma). a - "Calciolepersonnite” (Lps) enclosing vandendriesscheite (Van) and becquerelite (Bq). b - Detailed view on the fan-shaped aggregates of "calciolepersonnite" needle crystals. 
Tab. 5 Chemical composition of leesite-like mineral phase from Gemerská Poloma (empirical formulae calculated on the basis of $4 \mathrm{U}$ apfu and $20 \mathrm{O}$ )

\begin{tabular}{|c|c|c|c|c|}
\hline & 1 & 2 & 3 & Mean \\
\hline $\mathrm{Na}_{2} \mathrm{O}$ & 0.02 & 0.00 & 0.00 & 0.01 \\
\hline $\mathrm{K}_{2} \mathrm{O}$ & 2.50 & 2.89 & 2.34 & 2.58 \\
\hline $\mathrm{CaO}$ & 0.04 & 0.00 & 0.00 & 0.01 \\
\hline $\mathrm{SrO}$ & 0.05 & 0.06 & 0.04 & 0.05 \\
\hline $\mathrm{BaO}$ & 0.21 & 0.36 & 0.00 & 0.19 \\
\hline $\mathrm{FeO}$ & 0.21 & 0.02 & 0.19 & 0.14 \\
\hline $\mathrm{ZnO}$ & 0.05 & 0.12 & 0.11 & 0.09 \\
\hline $\mathrm{PbO}$ & 1.01 & 0.00 & 0.13 & 0.38 \\
\hline $\mathrm{Al}_{2} \mathrm{O}_{3}$ & 0.19 & 0.00 & 0.00 & 0.06 \\
\hline $\mathrm{SiO}_{2}$ & 0.18 & 0.00 & 0.00 & 0.06 \\
\hline $\mathrm{UO}_{3}$ & 86.98 & 90.40 & 82.63 & 86.67 \\
\hline $\mathrm{H}_{2} \mathrm{O}^{*}$ & 10.00 & 10.74 & 9.80 & 10.18 \\
\hline$\Sigma$ wt. $\%$ & 101.43 & 104.59 & 95.23 & 100.42 \\
\hline $\mathrm{Na}$ & 0.008 & 0.000 & 0.000 & 0.003 \\
\hline K & 0.699 & 0.777 & 0.689 & 0.721 \\
\hline $\mathrm{Ca}$ & 0.009 & 0.000 & 0.000 & 0.003 \\
\hline $\mathrm{Sr}$ & 0.006 & 0.008 & 0.005 & 0.006 \\
\hline $\mathrm{Ba}$ & 0.018 & 0.030 & 0.000 & 0.016 \\
\hline $\mathrm{Fe}$ & 0.038 & 0.003 & 0.036 & 0.026 \\
\hline $\mathrm{Zn}$ & 0.009 & 0.018 & 0.018 & 0.015 \\
\hline $\mathrm{Pb}$ & 0.060 & 0.000 & 0.008 & 0.023 \\
\hline $\mathrm{Al}$ & 0.050 & 0.000 & 0.000 & 0.017 \\
\hline$\Sigma$ A site & 0.895 & 0.836 & 0.755 & 0.829 \\
\hline $\mathrm{SiO}_{4}^{4-}$ & 0.038 & 0.000 & 0.000 & 0.013 \\
\hline $\mathrm{UO}_{2}{ }^{2+}$ & 4.000 & 4.000 & 4.000 & 4.000 \\
\hline Total & 4.934 & 4.836 & 4.755 & 4.842 \\
\hline $\mathrm{O}$ & 20.000 & 20.000 & 20.000 & 20.000 \\
\hline
\end{tabular}

$0.10 a p f u$ HREE); of the latter, the most important is $\mathrm{Tb}\left(0.26\right.$ wt. $\% \mathrm{~Tb}_{2} \mathrm{O}_{3} ; 0.07$ apfu $\left.\mathrm{Tb}\right)$. Regarding the other elements present at the cationic (A) site, the most significant is $\mathrm{Ca}(0.99-1.64$ wt. \% $\mathrm{CaO} ; 1.64-2.62$ apfu $\mathrm{Ca}$ ), which shows a strong positive correlation with $\mathrm{Y}+$ REE $(r=+0.59)$. Potassium content varies in the range $0.48-0.89$ wt. $\% \mathrm{~K}_{2} \mathrm{O}(0.47-0.86$ apfu K). Monovalent elements $(\mathrm{K}+\mathrm{Na})$ show a slight negative correlation with $\mathrm{Y}+\operatorname{REE}(r=-0.45), \mathrm{K}$ is weakly negatively correlated with $\mathrm{Ca}+\mathrm{Y}+\mathrm{REE}(r=-0.31)$. Thus, in this mineral phase, $\mathrm{Ca}$ and REE may be both partially substituted by $\mathrm{K}$. The content of other elements (Tab. 6) is insignificant and reaches only the first tenths of wt. \% of oxides. The presence of carbon (qualitative) was proven by the non-standardized EDS analysis. The average chemical composition of studied lepersonnite-like mineral can be expressed as: $\left(\mathrm{K}_{0.62} \mathrm{Na}_{0.09}\right)_{\Sigma 0.71}\left(\mathrm{Ca}_{2.08} \mathrm{Mg}_{0.04} \mathrm{Sr}_{0.02} \mathrm{Ba}_{0.02} \mathrm{Fe}_{0.05}\right.$ $\left.\mathrm{Zn}_{0.05} \mathrm{~Pb}_{0.03}\right)_{\Sigma 2.30}(\mathrm{Y}+\mathrm{REE})_{\Sigma 0.92}\left[\left(\mathrm{UO}_{2}\right)_{23.76}\left\{\left(\mathrm{SiO}_{4}\right)_{3.19}\left(\mathrm{PO}_{4}\right)_{0.11}\right.\right.$ $\left.\left.\left(\mathrm{AsO}_{4}\right)_{0.02}\left(\mathrm{SO}_{4}\right)_{0.02}\right\}_{53.34}\left(\mathrm{CO}_{3}\right)_{8}(\mathrm{OH})_{26.37}\right] \cdot 46.82 \mathrm{H}_{2} \mathrm{O}$. The studied unnamed mineral shows a deficiency in anionic content (an average of 0.66 apfu $\mathrm{Si}+\mathrm{P}+\mathrm{As}+\mathrm{S}$; Tab. 6), compared to the ideal composition of lepersonnite- $(\mathrm{Gd})$, $\mathrm{Ca}(\mathrm{REE})_{2}\left(\mathrm{UO}_{2}\right)_{24}\left(\mathrm{SiO}_{4}\right)_{4}\left(\mathrm{CO}_{3}\right)_{8}(\mathrm{OH})_{24} \cdot 48 \mathrm{H}_{2} \mathrm{O}$ (Deliens and Piret 1982).

\section{Discussion and conclusions}

\subsection{Notes on the chemical composition of vandendriesscheite and lepersonnite-like phases}

From the thirty known natural uranyl-oxide hydroxy-hydrates (28 mineral species stated in Plášil 2014, $2017+$ shinkolobweite; Olds et al. 2017b and kroupaite; Pláśil et al. 2017), ten contain $\mathrm{Pb}$ as a fundamental constituent (richetite, masuyite, spriggite, fourmarierite, meta-/ vandendriesscheite, sayrite, wölsendorfite, curite and shinkolobweite). Potassium, as an essential component, is present in four minerals only (agrinierite, compreignacite, rameauite and leesite).

In natural $\mathrm{Pb}$-rich uranyl-oxide hydroxy-hydrates, potassium is usually present only as an admixture; e.g., $\sim 0.25$ wt. $\% \mathrm{~K}_{2} \mathrm{O}$ were found in vandendriesscheite from Shinkolobwe, Congo (Finch 1994). More significant potassium contents have not yet been reported for vandendriesscheite. Until now, there are only two phases to contain both $\mathrm{Pb}$ and $\mathrm{K}$ as the essential constituents: gauthierite $\mathrm{KPb}\left[\left(\mathrm{UO}_{2}\right)_{7} \mathrm{O}_{5}(\mathrm{OH})_{7}\right] \cdot 8 \mathrm{H}_{2} \mathrm{O}$ (Olds et al. 2017a) and kroupaite $\mathrm{KPb}_{0,5}\left[\left(\mathrm{UO}_{2}\right)_{8} \mathrm{O}_{4}(\mathrm{OH})_{10}\right] \cdot 10 \mathrm{H}_{2} \mathrm{O}$ (Plášil et al. 2017). Both elements may also be present at significant amounts in calciouranoite.

Compared to the ideal vandendriesscheite $(\sim 10.2$ wt. \% $\mathrm{PbO} ; 1.5$ apfu $\mathrm{Pb})$, the $\mathrm{Pb}$ content in the mineral phase from the Gemerská Poloma is lower (average of 7.69 wt. \% $\mathrm{PbO} ; 1.2$ apfu $\mathrm{Pb}$; Tab. 4) and an average $\mathrm{K}_{2} \mathrm{O}$ content reaches 1.32 wt. \% (0.49 apfu K). The studied vandendriesscheite with its unusual chemical composition imitates a transition phase between gauthierite and vandendriesscheite (Fig. 6a-b) approaching that of the natural gauthierite (see electron-microprobe analysis in Olds et al. 2017a). But there cannot exist a series of solidsolutions with variable $\mathrm{K}: \mathrm{Pb}$ ratios between these minerals, due to their different crystal structure (see Burns 1997; Olds et al. 2017a). Negative correlation of K with $\mathrm{Pb}$ indicates that in the Gemerská Poloma vandendriesscheite, lead is partially replaced by potassium (and other cations). For the uranyl-oxide hydroxy-hydrates, this is unusual. For example, fourmarierite (general formula $\mathrm{Pb}_{1-\mathrm{x}}\left[\left(\mathrm{UO}_{2}\right) 4 \mathrm{O}_{3-2 \mathrm{x}}(\mathrm{OH})_{4+2 \mathrm{x}}\right] \cdot 4 \mathrm{H}_{2} \mathrm{O}$; $\mathrm{Li}$ and Burns 2000$)$ in which potassium is introduced into the cationic position $\left(0.70-1.56\right.$ wt. $\left.\% \mathrm{~K}_{2} \mathrm{O} ; 0.20-0.45 a p f u \mathrm{~K}\right)$ was found so far only in Jáchymov deposit, Czech Republic (Sejkora et al. 2013). 
Uranyl-oxide hydroxy-hydrates and "calciolepersonnite" from the Gemerská Poloma (Gemeric Unit, Slovakia)

Tab. 6 Chemical composition of “calciolepersonnite” from Gemerská Poloma (empirical formulae calculated on the basis of 31 apfu)

\begin{tabular}{|c|c|c|c|c|c|c|c|c|c|c|c|c|c|c|}
\hline & 1 & 2 & 3 & 4 & 5 & 6 & 7 & 8 & 9 & 10 & 11 & 12 & 13 & Mean \\
\hline $\mathrm{Na}_{2} \mathrm{O}$ & 0.08 & 0.06 & 0.16 & 0.00 & 0.02 & 0.06 & 0.09 & 0.01 & 0.01 & 0.06 & 0.13 & 0.04 & 0.05 & 0.06 \\
\hline $\mathrm{K}_{2} \mathrm{O}$ & 0.74 & 0.81 & 0.56 & 0.48 & 0.58 & 0.58 & 0.62 & 0.53 & 0.58 & 0.76 & 0.62 & 0.89 & 0.56 & 0.64 \\
\hline $\mathrm{MgO}$ & 0.00 & 0.04 & 0.02 & 0.02 & 0.04 & 0.03 & 0.00 & 0.02 & 0.00 & 0.03 & 0.00 & 0.00 & 0.01 & 0.02 \\
\hline $\mathrm{CaO}$ & 0.99 & 1.12 & 1.17 & 1.27 & 1.21 & 1.21 & 1.38 & 1.05 & 1.46 & 1.44 & 1.41 & 1.31 & 1.64 & 1.28 \\
\hline $\mathrm{SrO}$ & 0.05 & 0.00 & 0.06 & 0.00 & 0.00 & 0.03 & 0.00 & 0.03 & 0.10 & 0.00 & 0.00 & 0.06 & 0.02 & 0.03 \\
\hline $\mathrm{BaO}$ & 0.00 & 0.00 & 0.00 & 0.00 & 0.09 & 0.00 & 0.00 & 0.00 & 0.00 & 0.00 & 0.00 & 0.40 & 0.00 & 0.04 \\
\hline $\mathrm{FeO}$ & 0.00 & 0.04 & 0.00 & 0.13 & 0.00 & 0.00 & 0.12 & 0.00 & 0.10 & 0.02 & 0.15 & 0.00 & 0.00 & 0.04 \\
\hline $\mathrm{ZnO}$ & 0.05 & 0.12 & 0.00 & 0.18 & 0.00 & 0.08 & 0.07 & 0.00 & 0.00 & 0.00 & 0.00 & 0.00 & 0.05 & 0.04 \\
\hline $\mathrm{PbO}$ & 0.11 & 0.00 & 0.00 & 0.00 & 0.00 & 0.00 & 0.00 & 0.05 & 0.00 & 0.20 & 0.04 & 0.43 & 0.08 & 0.07 \\
\hline $\mathrm{Y}_{2} \mathrm{O}_{3}$ & 0.02 & 0.08 & 0.03 & 0.01 & 0.00 & 0.00 & 0.03 & 0.05 & 0.03 & 0.02 & 0.05 & 0.00 & 0.00 & 0.02 \\
\hline $\mathrm{La}_{2} \mathrm{O}_{3}$ & n.a. & n.a. & n.a. & n.a. & n.a. & n.a. & n.a. & 0.05 & 0.03 & 0.00 & 0.01 & 0.10 & 0.00 & 0.02 \\
\hline $\mathrm{Ce}_{2} \mathrm{O}_{3}$ & n.a. & n.a. & n.a. & n.a. & n.a. & n.a. & n.a. & 0.21 & 0.19 & 0.14 & 0.20 & 0.14 & 0.16 & 0.08 \\
\hline $\mathrm{Pr}_{2} \mathrm{O}_{3}$ & n.a. & n.a. & n.a. & n.a. & n.a. & n.a. & n.a. & 0.18 & 0.18 & 0.02 & 0.07 & 0.05 & 0.00 & 0.04 \\
\hline $\mathrm{Nd}_{2} \mathrm{O}_{3}$ & 0.92 & 0.71 & 1.09 & 1.30 & 1.07 & 1.17 & 1.21 & 1.06 & 1.20 & 1.03 & 1.04 & 0.94 & 1.19 & 1.07 \\
\hline $\mathrm{Sm}_{2} \mathrm{O}_{3}$ & 1.09 & 1.07 & 1.51 & 1.16 & 1.27 & 1.39 & 1.47 & 1.48 & 1.56 & 1.28 & 1.55 & 1.11 & 1.37 & 1.33 \\
\hline $\mathrm{Eu}_{2} \mathrm{O}_{3}$ & n.a. & n.a. & n.a. & n.a. & n.a. & n.a. & n.a. & 0.11 & 0.13 & 0.12 & 0.16 & 0.10 & 0.15 & 0.06 \\
\hline $\mathrm{Gd}_{2} \mathrm{O}_{3}$ & 0.52 & 0.39 & 0.20 & 0.52 & 0.51 & 0.47 & 0.50 & 0.40 & 0.52 & 0.43 & 0.45 & 0.47 & 0.56 & 0.46 \\
\hline $\mathrm{Tb}_{2} \mathrm{O}_{3}$ & 0.20 & 0.29 & 0.36 & 0.26 & 0.32 & 0.22 & 0.25 & 0.23 & 0.15 & 0.17 & 0.29 & 0.34 & 0.30 & 0.26 \\
\hline $\mathrm{Dy}_{2} \mathrm{O}_{3}$ & 0.18 & 0.12 & 0.02 & 0.00 & 0.00 & 0.00 & 0.10 & 0.03 & 0.11 & 0.09 & 0.04 & 0.10 & 0.06 & 0.06 \\
\hline $\mathrm{Tm}_{2} \mathrm{O}_{3}$ & n.a. & n.a. & n.a. & n.a. & n.a. & n.a. & n.a. & 0.12 & 0.07 & 0.11 & 0.06 & 0.15 & 0.09 & 0.05 \\
\hline $\mathrm{Yb}_{2} \mathrm{O}_{3}$ & n.a. & n.a. & n.a. & n.a. & n.a. & n.a. & n.a. & 0.03 & 0.16 & 0.04 & 0.11 & 0.11 & 0.22 & 0.05 \\
\hline $\mathrm{SiO}_{2}$ & 1.95 & 1.94 & 2.26 & 2.10 & 2.20 & 2.21 & 2.24 & 2.12 & 2.30 & 2.03 & 2.10 & 1.84 & 2.11 & 2.11 \\
\hline $\mathrm{P}_{2} \mathrm{O}_{5}$ & 0.00 & 1.39 & 0.02 & 0.05 & 0.04 & 0.03 & 0.02 & 0.01 & 0.00 & 0.04 & 0.03 & 0.52 & 0.00 & 0.17 \\
\hline $\mathrm{As}_{2} \mathrm{O}_{5}$ & 0.16 & 0.14 & 0.05 & 0.00 & 0.01 & 0.02 & 0.00 & 0.00 & 0.06 & 0.02 & 0.00 & 0.06 & 0.02 & 0.04 \\
\hline $\mathrm{SO}_{3}$ & 0.03 & 0.07 & 0.04 & 0.01 & 0.00 & 0.02 & 0.01 & 0.01 & 0.01 & 0.01 & 0.00 & 0.02 & 0.01 & 0.02 \\
\hline $\mathrm{UO}_{3}$ & 75.24 & 75.02 & 72.84 & 74.40 & 73.81 & 76.71 & 74.72 & 73.73 & 75.33 & 74.53 & 75.71 & 74.15 & 74.66 & 74.68 \\
\hline $\mathrm{CO}_{2}^{*}$ & 4.80 & 4.76 & 4.77 & 4.77 & 4.78 & 4.78 & 4.75 & 4.73 & 4.85 & 4.82 & 4.82 & 4.82 & 4.85 & 4.79 \\
\hline $\mathrm{H}_{2} \mathrm{O}^{*}$ & 14.74 & 14.60 & 14.63 & 14.64 & 14.66 & 14.68 & 14.58 & 14.52 & 14.88 & 14.81 & 14.81 & 14.81 & 14.88 & 14.71 \\
\hline$\Sigma$ wt. $\%$ & 101.85 & 102.77 & 99.80 & 101.32 & 100.61 & 103.68 & 102.17 & 100.73 & 104.00 & 102.21 & 103.82 & 102.97 & 103.04 & 102.23 \\
\hline $\mathrm{Na}$ & 0.123 & 0.085 & 0.246 & 0.000 & 0.030 & 0.091 & 0.134 & 0.018 & 0.007 & 0.089 & 0.180 & 0.051 & 0.072 & 0.087 \\
\hline $\mathrm{K}$ & 0.728 & 0.773 & 0.553 & 0.471 & 0.570 & 0.551 & 0.595 & 0.529 & 0.542 & 0.728 & 0.584 & 0.855 & 0.530 & 0.616 \\
\hline $\mathrm{Mg}$ & 0.005 & 0.078 & 0.046 & 0.048 & 0.099 & 0.056 & & 0.044 & & 0.077 & & 0.007 & 0.024 & 0.037 \\
\hline $\mathrm{Ca}$ & 1.644 & 1.787 & 1.948 & 2.082 & 2.005 & 1.932 & 2.220 & 1.754 & 2.314 & 2.328 & 2.239 & 2.113 & 2.619 & 2.076 \\
\hline $\mathrm{Sr}$ & 0.048 & & 0.055 & & & 0.024 & & 0.025 & 0.083 & & & 0.052 & 0.017 & 0.023 \\
\hline $\mathrm{Ba}$ & & 0.000 & & & 0.051 & & & & & 0.000 & 0.000 & 0.237 & & 0.022 \\
\hline $\mathrm{Fe}$ & & 0.049 & & 0.168 & & & 0.154 & & 0.120 & 0.024 & 0.184 & & & 0.054 \\
\hline $\mathrm{Zn}$ & 0.054 & 0.131 & & 0.207 & 0.000 & 0.090 & 0.082 & & & & & & 0.060 & 0.048 \\
\hline $\mathrm{Pb}$ & 0.047 & & & & & & & 0.019 & & 0.082 & 0.016 & 0.173 & 0.033 & 0.029 \\
\hline $\mathrm{Y}$ & 0.007 & 0.031 & 0.011 & 0.004 & & & 0.010 & 0.019 & 0.012 & 0.006 & 0.019 & & & 0.009 \\
\hline $\mathrm{La}$ & & & & & & & & 0.015 & 0.009 & 0.001 & 0.002 & 0.027 & & 0.004 \\
\hline $\mathrm{Ce}$ & & & & & & & & 0.060 & 0.052 & 0.038 & 0.055 & 0.039 & 0.044 & 0.022 \\
\hline $\operatorname{Pr}$ & & & & & & & & 0.050 & 0.049 & 0.007 & 0.020 & 0.014 & & 0.011 \\
\hline $\mathrm{Nd}$ & 0.253 & 0.190 & 0.302 & 0.356 & 0.294 & 0.313 & 0.323 & 0.295 & 0.316 & 0.276 & 0.277 & 0.252 & 0.319 & 0.290 \\
\hline $\mathrm{Sm}$ & 0.289 & 0.275 & 0.403 & 0.305 & 0.339 & 0.356 & 0.379 & 0.398 & 0.397 & 0.333 & 0.396 & 0.287 & 0.354 & 0.347 \\
\hline $\mathrm{Eu}$ & & & & & & & & 0.030 & 0.034 & 0.031 & 0.040 & 0.027 & 0.037 & 0.015 \\
\hline $\mathrm{Gd}$ & 0.133 & 0.096 & 0.052 & 0.131 & 0.130 & 0.117 & 0.125 & 0.103 & 0.127 & 0.107 & 0.110 & 0.116 & 0.138 & 0.114 \\
\hline $\mathrm{Tb}$ & 0.051 & 0.072 & 0.090 & 0.065 & 0.082 & 0.055 & 0.062 & 0.058 & 0.037 & 0.043 & 0.071 & 0.085 & 0.073 & 0.065 \\
\hline Dy & 0.045 & 0.029 & 0.004 & & & & 0.025 & 0.007 & 0.025 & 0.021 & 0.009 & 0.025 & 0.014 & 0.016 \\
\hline $\mathrm{Tm}$ & & & & & & & & 0.029 & 0.016 & 0.025 & 0.013 & 0.036 & 0.020 & 0.011 \\
\hline $\mathrm{Yb}$ & & & & & & & & 0.007 & 0.035 & 0.009 & 0.025 & 0.024 & 0.051 & 0.012 \\
\hline$\Sigma$ A site & 3.425 & 3.596 & 3.710 & 3.836 & 3.600 & 3.585 & 4.109 & 3.459 & 4.176 & 4.226 & 4.239 & 4.420 & 4.406 & 3.910 \\
\hline$\Sigma \mathrm{Y}+\mathrm{REE}$ & 0.778 & 0.694 & 0.861 & 0.860 & 0.845 & 0.841 & 0.925 & 1.070 & 1.110 & 0.898 & 1.037 & 0.933 & 1.050 & 0.915 \\
\hline $\mathrm{SiO}_{4}^{4-}$ & 3.012 & 2.898 & 3.504 & 3.217 & 3.403 & 3.297 & 3.351 & 3.306 & 3.400 & 3.069 & 3.113 & 2.772 & 3.146 & 3.191 \\
\hline $\mathrm{PO}_{4}^{3-}$ & & 0.874 & 0.015 & 0.034 & 0.029 & 0.016 & 0.010 & 0.009 & 0.001 & 0.022 & 0.018 & 0.331 & & 0.105 \\
\hline $\mathrm{AsO}_{4}^{3-}$ & 0.065 & 0.053 & 0.021 & & 0.004 & 0.008 & & & 0.023 & 0.007 & & 0.024 & 0.007 & 0.016 \\
\hline $\mathrm{SO}_{4}^{2-}$ & 0.034 & 0.081 & 0.050 & 0.015 & & 0.026 & 0.011 & 0.014 & 0.012 & 0.016 & 0.002 & 0.026 & 0.010 & 0.023 \\
\hline$\Sigma$ T site & 3.111 & 3.905 & 3.590 & 3.266 & 3.435 & 3.346 & 3.371 & 3.329 & 3.436 & 3.114 & 3.133 & 3.153 & 3.163 & 3.335 \\
\hline $\mathrm{UO}_{2}^{2+}$ & 24.464 & 23.489 & 23.700 & 23.898 & 23.958 & 24.069 & 23.520 & 24.211 & 23.388 & 23.643 & 23.617 & 23.427 & 23.431 & 23.755 \\
\hline $\mathrm{CO}_{3}^{2-*}$ & 8.000 & 8.000 & 8.000 & 8.000 & 8.000 & 8.000 & 8.000 & 8.000 & 8.000 & 8.000 & 8.000 & 8.000 & 8.000 & 8.000 \\
\hline $\mathrm{OH}^{* *}$ & 27.393 & 23.491 & 24.658 & 26.855 & 25.667 & 26.197 & 26.001 & 26.582 & 25.994 & 27.460 & 27.497 & 27.515 & 27.495 & 26.370 \\
\hline $\mathrm{H}_{2} \mathrm{O}^{* *}$ & 46.303 & 48.254 & 47.671 & 46.573 & 47.167 & 46.901 & 47.000 & 46.709 & 47.003 & 46.270 & 46.251 & 46.243 & 46.253 & 46.815 \\
\hline
\end{tabular}

$\mathrm{CO}_{3}^{*}$ and $\mathrm{H}_{2} \mathrm{O}^{*}$ - calculation based on the theoretical content of $8 \mathrm{CO}_{3}$ and $60 \mathrm{H}_{2} \mathrm{O}$ in ideal lepersonnite formula

$\mathrm{OH}^{* *}$ - calculation based on the charge-balance (at the expense of $60 \mathrm{H}_{2} \mathrm{O}$, resulting in reduced $\mathrm{H}_{2} \mathrm{O}$ content)

n.a. - not analysed 


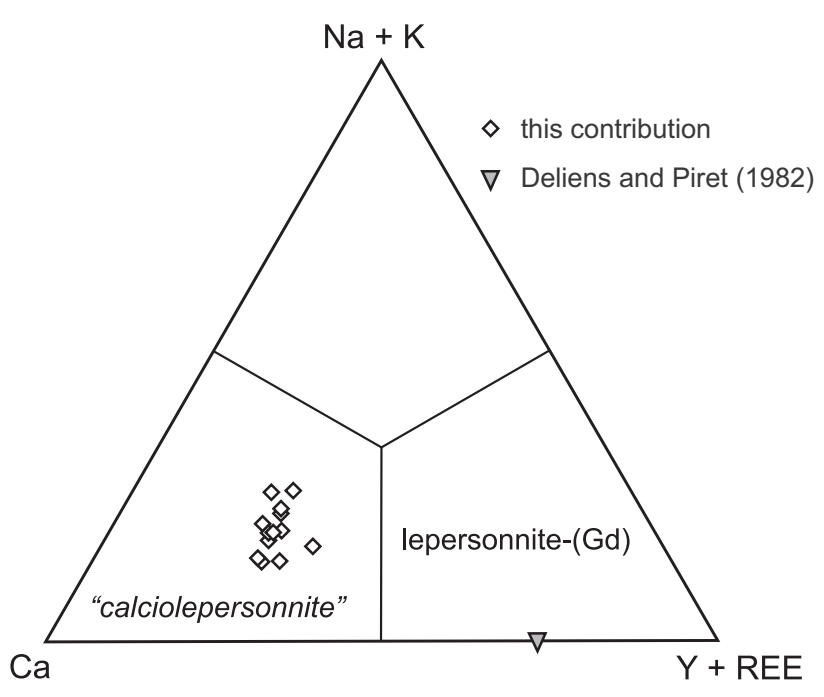

Fig. 9 Compositions of “calciolepersonnite” from Gemerská Poloma compared with those of lepersonnite-(Gd) in ternary $\mathrm{Ca}-\mathrm{Na}+\mathrm{K}-\mathrm{Y}+$ REE diagram (apfu).

Lepersonnite $\left[\mathrm{Ca}(\mathrm{REE})_{2}\left(\mathrm{UO}_{2}\right)_{24}\left(\mathrm{SiO}_{4}\right)_{4}\left(\mathrm{CO}_{3}\right)_{8}(\mathrm{OH})_{24}\right.$. $48 \mathrm{H}_{2} \mathrm{O}$ ], a mixed uranyl carbonate-silicate, was described from the Shinkolobwe deposit, Congo (Deliens and Piret 1982). According to the main element in the cationic (A) position, its name was modified to lepersonnite-(Gd). Shinkolobwe until now remains the only locality with the occurrence of this rare mineral. The chemical composition of unnamed uranyl carbonate-silicate from Gemerská Poloma (Tab. 6) is notably similar to lepersonnite(Gd), but occupancy of the cationic position is distinct. Compared to the ideal formula of lepersonnite-(Gd), the $\mathrm{REE}$ are lower and $\mathrm{Ca}$ higher; furthermore, there are monovalent cations (especially $\mathrm{K}$ ) present. In the studied mineral, the ratios $M^{+}: \mathrm{Ca}:(\mathrm{Y}+\mathrm{REE})^{3+}$ and $M^{+}: M^{2+}:(\mathrm{Y}$ $+\mathrm{REE}^{3+}($ apfu $)$ are $1: 2.95: 1.30$ and $1: 3.26: 1.30$, respectively (Fig. 9). Thus, occupancy of the cationic (A) position suggests that the mineral phase from Gemerská Poloma could represent a calcium-dominant member of a broader mineral group, containing also lepersonnite-(Gd). According to the principal element in the cationic position, it can be preliminarily named "calciolepersonnite", with an ideal formula $\mathrm{KCa}_{2}(\mathrm{REE})\left(\mathrm{UO}_{2}\right)_{24}\left(\mathrm{SiO}_{4}\right)_{4}\left(\mathrm{CO}_{3}\right)_{8}$ $(\mathrm{OH})_{24} \cdot 48 \mathrm{H}_{2} \mathrm{O}$. Significant positive correlations of $\mathrm{Ca}$ with $\mathrm{Y}+\mathrm{REE}(r=0.59)$ and $\mathrm{K}+\mathrm{Na}$ (Fig. 9) indicate that it could also be a completely different mineral, not related to lepersonnite-(Gd).

\subsection{Origin of the uranyl oxide-hydroxy- -hydrates and "calciolepersonnite"}

Up to now, a considerable research has been focused on the weathering processes of uranium mineralization under oxidation conditions. The evolution and classification of supergene changes of the primary $\mathrm{U}^{\mathrm{IV}}$ minerals has been summarized, for instance, in Ščerbina (1963), Belova (1975, 2000), Krivovichev and Plášil (2013) or Plášil (2014). Primary UV minerals are, at the very initial stage of their supergene weathering, replaced by ianthinite (presence of $\mathrm{U}^{4+}$ and $\mathrm{U}^{6+}$ in structure), and then by uranyl-oxide hydroxy-hydrates (schoepite, fourmarierite, becquerelite, vandendriesscheite etc.); uranyl silicates follow later.

The formation of all above secondary minerals takes place under alkaline to neutral conditions. They are suitable for the mobilization/transport of released uranium in the form of easily soluble mono-, di- and tricarbonate uranyl complexes (Langmuir 1978). Uranyl carbonates usually precipitate by evaporation of alkaline solutions at relatively high $\mathrm{CO}_{2}$ fugacity (Finch and Murakami 1999), although they may also originate in a slightly acidic environment (sensu the scheme in Krivovichev and Plášil 2013).

Sulphide minerals form a rather broad association in quartz \pm apatite veins with $\mathrm{U}, \mathrm{REE}$ and Au mineralization in Lower Palaeozoic rocks of the Gemeric Unit. Still, they are usually fine-grained and scattered, and only rarely they occur in larger accumulations (first centimetres in size) (Novotný and Čížek 1979; Rojkovič and Novotný 1993; Rojkovič et al. 1995, 1997 1999; Novotný et al. 1999; Števko et al. 2014). As for uranium supergene minerals, the most abundant are uranyl arsenates/phosphates of the autunite group (uranium micas): autunite, torbernite, zeunerite, trögerite and probably kahlerite (Rojkovič 1997; Rojkovič et al. 1997, 1999). These minerals are typically formed under acidic conditions of weathering (Krivovichev and Plášil 2013 and references therein).

An analogous situation also occurs in the J-1 vein at Gemerská Poloma, from which comes the studied sample. Of the broad group of uranyl-oxide hydroxy-hydrates, only fourmarierite has been known from the studied site (confirmed by XRD analysis; Ferenc et al. 2003). Studied gangue is composed exclusively of brannerite and a small amount of quartz and uraninite, without sulphides. It represents exceptional accumulation of brannerite, which otherwise forms only small clusters of columnar crystals (up to $0.5 \mathrm{~cm}$ long) in the vein quartz. The association of uranyl-oxide hydrate-hydrates (becquerelite, leesite-like mineral, vandendriesscheite), which are accompanied by the younger mixed unnamed uranyl carbonate-silicate, "calciolepersonnite", has been identified in the yellow crusts on its surface. This mineral association indicates an alkaline to neutral environment of primary uranium (U ${ }^{\mathrm{IV}}$ ) weathering, due to the minimal content of sulphides in the gangue.

Based on the current results and field observations, three evolutionary stages of supergene mineral association can be described at the J-1 vein at Gemerská Poloma (following Finch and Ewing 1992 or Plášil et al. 2014): 
(I) uranyl-oxide hydroxy-hydrates (fourmarierite, becquerelite, leesite-like phase, vandendriesscheite) partially or completely replace $\mathrm{U}^{\mathrm{IV}}$ minerals; (II) an unnamed uranyl carbonate-silicate mineral ("calciolepersonnite") replaces uranyl-oxide hydroxy-hydrates, apparently indicating a shift towards relatively acid conditions and (III) uranyl phosphates/arsenates of the autunite group form [autunite, torbernite, zeunerite, trögerite (?), kahlerite (?)]. Relative to uranyl-oxide hydroxy-hydrates, these minerals precipitate relatively far from the accumulations of primary $\left(\mathrm{U}^{\mathrm{IV}}\right)$ minerals (cracks and cavities in the gangue or in the surrounding non-mineralized rocks). Their origin documents the change of alkaline neutral to acidic environment, the latter due the more advanced weathering of vein sulphides (releasing the sulphuric acid).

The average $\mathrm{Y}+\mathrm{REE}$ content in quartz veins containing U mineralization (Krátka Dolina-Peklisko occurrences) is $770 \mathrm{ppm}$ (Rojkovič 1997). Since minerals such as monazite or xenotime are not abundant in the J-1vein, most of the Y + REE required for "calciolepersonnite" formation were probably released from the host rocks (Göb et al. 2013) and not from the primary, hydrothermal uranium or REE minerals. This is supported by the absence of Y + REE in older uranyl-oxide hydroxy-hydrates that directly replace brannerite.

Acknowledgements. This work was supported by the Slovak Research and Development Agency under the contract APVV-15-0050, as well as the Ministry of Education, Slovak Republic VEGA-1/0237/18 and VEGA $-2 / 0023 / 17$ projects. Authors thank to Travis A. Olds, Jakub Plášil and Martin Števko for precise reviews and improvement of the manuscript. Finally, we thank to Jiří Sejkora (Handling editor) and Vojtěch Janoušek (Editorin-Chief) for their constructive suggestions.

\section{References}

Bajaní $\breve{S}$, Hanzel V, Mello J, Pristaš J, Reichwalder P, SNOPKo L, VozÁR J, VozÁRovÁ A (1983) Explanation to geological map of the Slovenské Rudohorie Mts. eastern part, $1: 50$ 000. State Geological Institute of D. Štúr, Bratislava, pp 1-223 (in Slovak)

BAJANÍK Š, IVANičKa J, MELlo J, ReICHWALdER P, PRistaš J, Snopko L, VozÁr J, VozÁrová A (1984) Geological map of the Slovenské Rudohorie Mts. - eastern part, 1 : 50 000. State Geological Institute of D. Stúr, Bratislava (in Slovak)

Bartlett JR, CoONey RP (1989) On the determination of uranium-oxygen bond lengths in dioxouranium (VI) compounds by Raman spectroscopy. J Mol Struct 193: 295-300
Belova LN (1975) Zones of Oxidation of Hydrothermal Uranium Deposits. Nedra, Moscow, pp 1-173 (in Russian)

BELOVA LN (2000) Formation conditions of oxidation zones of uranium deposits and uranium mineral accumulations in the gipergenesis zone. Geol Ore Dep 42: 103-110

Billiet V, DE Jong WF (1935) Schoepite and becquerelite. Naturw Tijdschr Ned-Indië 17: 157-162 (in Dutch)

Bruker AXS (2010) Difrac.Eva - User Manual. Bruker AXS, Karlsruhe, pp 1-134

BuRns PC (1997) A new uranyl oxide hydrate sheet in vandendriesscheite: implications for mineral paragenesis and the corrosion of spent nuclear fuel. Amer Miner 82: 1176-1186

BuRns PC, Li Y (2002) The structures of becquerelite and Sr-exchanged becquerelite. Amer Miner 87: 550-557

Christ CL, Clark JR (1960) Crystal chemical studies of some uranyl oxide hydrates. Amer Miner 45: 1026-1061

Čejka J, Sejkora J, Skála R, Čejka J, Novotná M, Ederová J (1998) Contribution to the crystal chemistry of synthetic becquerelite, billietite and protasite. Neu Jb Mineral, Abh 174: 159-180

Deliens M, Piret P (1982) Bijvoetite et lepersonnite, carbonates hydratés d'uranyle et de terres rares de Shinkolobwe, Zaire. Canad Mineral 20: 231-238

Donát A, Minál F, Novotný L (2000) Geological-Exploration Works on the Au in Lower Paleozoic of SpišskoGemerské Rudohorie Mts. Unpublished Report, State Geological Institute of D. Štúr, Bratislava, pp 1-209 (in Slovak)

Downs RT, Hall-Wallace M (2003) The American Mineralogist Crystal Structure Database. Amer Miner 88: $247-250$

FARYAD SW (1991a) Metamorphism of the Early Paleozoic salic to intermediate volcanic rocks. Miner Slov 23: 325-332 (in Slovak)

FARYAD SW (1991b) Metamorphism of the Early Paleozoic sedimentary rocks in Gemericum. Miner Slov 23: 315-324 (in Slovak)

FERENC Š, RoJKoviČ I, MAŤo L' (2003) Uranyl minerals of Western Carpathians. In: ZıMÁK J (ed) Mineralogy of Bohemian Massif and Western Carpathians. Palacký University, Olomouc, pp 17-23 (in Slovak)

FINCH JR (1994) Paragenesis and Crystal Chemistry of the Uranyl Oxide Hydrates. Unpublished PhD. Thesis, University of New Mexico, Albuquerque, pp 1-257

FINCH JR, EwING RC (1992) The corrosion of uraninite under oxidizing conditions. J Nucl Mater 190: 133-156

Finch R, MuraKami T (1999) Systematics and paragenesis of uranium minerals. In: BuRns PC, FINCH R (eds) Uranium: Mineralogy, Geochemistry and the Environment. Mineralogical Society of America Reviews in Mineralogy 38: pp 91-179 
Frondel JW, CutTitTA F (1953) Studies of uranium minerals (XII); The status of billietite and becquerelite. Amer Miner 38: 1019-1024

Frost RL, ČEJKA J, WeIER ML (2007) Raman spectroscopic study of the uranyl oxyhydroxide hydrates: becquerelite, billietite, curite, schoepite and vandendriesscheite. J Raman Spectrosc 38: 460-466

Göв S, GÜHRING JE, BAU M, MARKL G (2013) Remobilization of $U$ and REE and the formation of secondary minerals in oxidized U deposits. Amer Miner 98: 530-548

Grecula P, KobulskÝ J, GAZDAČKo L, NÉMETH Z, HraŠKo L', Novotný L, Maglay J (2009) Geological map of the Spišsko-Gemerské Rudohorie Mts., 1:50 000. State Geological Institute of D. Štúr, Bratislava (in Slovak)

Grecula P, KobulskÝ J, GaZdAČKo L', NÉMETh Z, HraŠKo L', Novotný L, Maglay J, Pramuka S, Radvanec M, KuCHARIČ L, BAJTOŠ P, ZÁHOROVÁ L' (2011) Explanations to geological map of the Spišsko-Gemerské Rudohorie Mts., 1 : 50 000. State Geological Institute of D. Štúr, Bratislava, pp 1-308 (in Slovak)

Holland TJB, REDFERn SAT (1997) Unit cell refinement from powder diffraction data: the use of regression diagnostics. Mineral Mag 61: 65-77

IVANiČKa J, SNOPKo L, SNOpková P, VozÁrová A (1989) Gelnica Group - Lower Unit of Spišsko-Gemerské Rudohorie Mts. (West Carpathians), Early Paleozoic. Geol Zbor Geol Carpath 40: 483-501

Kohút M, Stein H, Uher P, Aimmerman A (2013) Re-Os and $\mathrm{U}-\mathrm{Th}-\mathrm{Pb}$ dating of the Rochovce granite and its mineralization (Western Carpathians, Slovakia). Geol Carpath 64: 71-79

Krivovichev SV, Pléšsl J (2013) Mineralogy and crystallography of uranium. In: Burns PC, Sigmon GE (eds) Uranium: From Cradle to Grave, Mineralogical Association of Canada Short Courses 43: pp 15-119

LANGMUIR D (1978) Uranium solution-mineral equilibria at low temperatures with applications to sedimentary ore deposits. Geochim Cosmochim Acta 42: 547-569

LI Y, BuRNs PC (2000) Investigation of crystal-chemistry variability in lead uranyl oxide hydrates II. Fourmarierite. Canad Mineral 38: 737-749

LiBowitzKy E (1999) Correlation of O-H stretching frequencies and $\mathrm{O}-\mathrm{H} \cdots \mathrm{O}$ hydrogen bond lengths in minerals. Monatsh Chem 130: 1047-1059

Mahel M, VozÁR J (1971) Contribution to knowledge of Permian and Triassic in the North-Gemeric syncline. Geol Práce, Spr 56: 47-66 (in Slovak)

Németh Z, Putiš M, Grecula P (2000) Tectonic evolution of Gemericum (the Western Carpathians) outlined by the results of petrotectonic research. Miner Slov 32: 169-172

Novotný L, ČížEK P (1979) New occurrence of uraniumgold mineralization to the south of Prakovce in SpišskoGemerské Rudohorie Mts. Miner Slov 11: 188-190 (in Slovak)
Novotný L, Háber M, KrižÁni I, RoJKovič I, Mihá́ F (1999) Gold in the Early Paleozoic rocks in the central part of the Spišsko-Gemerské Rudohorie Mts. Miner Slov 31: 211-216 (in Slovak)

Olds TA, Plášil J, Kampf AR, ŠKoda R, Burns PC, ČeJKa J, Bourgoin V, Boulliard JC (2017a) Gauthierite, $\mathrm{KPb}\left[\left(\mathrm{UO}_{2}\right)_{7} \mathrm{O}_{5}(\mathrm{OH})_{7}\right] \cdot 8 \mathrm{H}_{2} \mathrm{O}$, a new uranyl-oxide hydroxy-hydrate mineral from Shinkolobwe with a novel uranyl-anion sheet-topology. Eur J Mineral 29: 129-141

Olds TA, Lussier AJ, Oliver AG, Petříček V, PlášIl J, Kampf AR, Burns PC, Dembowski M, CARson SM, SteELE IM (2017b) Shinkolobweite, IMA 2016-095. CNMNC Newsletter No. 36, April 2017, p 404; Mineral Mag 81: 403-409

Olds TA, PlášIl J, Kampf AR, SPANo T, Haynes P, CARlson SM, Burns PC, Simonetti A, Mills OP (2018) Leesite $\mathrm{K}\left(\mathrm{H}_{2} \mathrm{O}\right)_{2}\left[\left(\mathrm{UO}_{2}\right)_{4} \mathrm{O}_{2}(\mathrm{OH})_{5}\right] \cdot 3 \mathrm{H}_{2} \mathrm{O}$, a new $\mathrm{K}$-bearing schoepite-family mineral from the Jomac mine, San Juan County, Utah, U.S.A. Amer Miner 103: 143-150

Pagoaga MK, Appleman DE, Stewart JM (1987) Crystal structures and crystal chemistry of the uranyl oxide hydrates becquerelite, billietite, and protasite. Amer Miner 72: $1230-1238$

Piret-Meunier J, Piret P (1982) Nouvelle determination de la structure cristalline de la becquerelite. Bull Minéral 105: 606-610

PLÁŠIL J (2014) Oxidation-hydration weathering of uraninite: the current state-of-knowledge. J Geosci 59: 99-114

PLÁŠIL J (2017) Uranyl-oxide hydroxy-hydrate minerals: their structural complexity and evolution trends. Eur J Mineral 30: 1-15

PláŠIL J, SEJKoRA J, ŠKoda R, ŠKÁcha P (2014) The recent weathering of uraninite from Červená vein, Jáchymov (Czech Republic): a fingerprint of the primary mineralization onto the alteration association. J Geosci 59: 223-253

PláŠIl J, KampF AR, Olds TA, SEJKORA J, ŠKoda R, BuRnS PC, ČEJKA J (2017) Kroupaite, IMA 2017-031. CNMNC Newsletter No. 38, August 2017, p 1036; Mineral Mag 81: 1033-1038

Poller U, Uher P, Janák M, Plašienka D, Kohút M (2001) Late Cretaceous age of the Rochovce Granite, Western Carpathians, constrained by U-Pb single-zircon dating in combination with cathodoluminescence imaging. Geol Carpath 52: 41-47

Protas J (1957) Propriétés et synthèse d'un oxyde hydraté d'uranium et de calcium de Shinkolobwe, Katanga. C R Acad Sci Paris 244: 91-93

Putiš M, Sergeev S, Ondrejka M, Larionov A, Siman P, Spišiak J, Uher P, PAderin I (2008) Cambrian-Ordovician metaigneous rocks associated with Cadomian fragments in the West-Carpathian basement dated by SHRIMP on zircons: a record from the Gondwana active margin setting. Geol Carpath 59: 3-18 
Radvanec M, Grecula P (2016) Geotectonic and metallogenetic evolution of Gemericum (inner Western Carpathians) from Ordovician to Jurassic. Miner Slov 48: 105-118

Radvanec M, KoneČnÝ P, OndreJka M, Putiš M, Uher P, NÉMETH Z (2009) The Gemeric granites as an indicator of the crustal extension above the Late-Variscan subduction zone during the Early Alpine riftogenesis (Western Carpathians): an interpretation from the monazite and zircon ages dates by CHIME and SHRIMP methods. Miner Slov 41: 381-394

RoJkovič I (1997) Uranium Mineralization in Slovakia. Comenius University, Bratislava, pp 1-117

RoJkovič I, NovotnÝ L (1993) Uranium mineralization in Gemericum. Miner Slov 25: 368-370 (in Slovak)

RoJkovič I, PušKeloví L', KHun M, MedveĎ J (1995) UREE-Au in veins and black shales of the Gemericum, Slovakia. In: PAŠAVA J, Ǩ̌́́BEK B, ŽÁK K (eds) Mineral Deposits: From Their Origin to Their Environmental Impacts. Balkema, Rotterdam, pp 789-792

Rojkovič I, HÁber M, Novotný L (1997) U-Au-CoBi-REE mineralization in the Gemeric Unit (Western Carpathians, Slovakia). Geol Carpath 48: 303-313

RoJkovič I, KonečnÝ P, Novotný L, PušKelová L', STREŠKO V (1999) Quartz-apatite-REE vein mineralization in Early Paleozoic rocks of the Gemeric Superunit, Slovakia. Geol Carpath 50: 215-227

SeJKora J, PlášIl J, Bureš B (2013) Unusual association of supergene uranium minerals from the Jan Evangelista vein, Jáchymov (Czech Republic). Bull mineral-petrolog Odd Nár Muz (Praha) 21: 143-156 (in Czech)

SnOPKo L, IvaničKa J (1978) Considerations on the paleogeography in the Lower Paleozoic of Spišsko-Gemerské
Rudohorie Mts. In: VozÁr J (ed) Paleogeographical Evolution of the Western Carpathians. Geological Institution of D. Štúr, Bratislava, pp 269-280 (in Slovak)

ŠČERBINA VV (1963) Geochemistry of uranium in the oxidation zone. In: Vinogradov AP (ed.) Basic Features of Uranium Geochemistry. Publishing House of the AS USSR, Moscow, pp 220-237 (in Russian)

ŠteVko M, Uher P, Ondrejka M, Ozdín D, BačíK P (2014) Quartz-apatite-REE phosphates-uraninite vein mineralization near Čučma (eastern Slovakia): a product of early Alpine hydrothermal activity in the Gemeric Superunit, Western Carpathians. J Geosci 59: 209-222

VAES JF (1947) Six noveaux minéraux d'urane provenant de Shinkolobwe (Katanga). Ann Soc géol Belg 70: 212-225

VARČEK C (1977) Some rare mineralization types in the Spišsko-Gemerské Rudohorie Mts. In: HÁBER M. (ed) Ore-Forming Processes in the Western Carpathians. Universitas Comeniana, Bratislava, pp 93-99 (in Slovak)

VozÁroví A (1993) Variscan metamorphism and crustal evolution of the Gemericum. Západ Karpaty, Sér Mineral Petrogr Geochém Metalogen 16: 55-117 (in Slovak)

Vozárová A, Šarinová K, Sergeev S, Larionov A, Presnyakov S (2010) Late Cambrian/Ordovician magmatic arc type volcanism in the Southern Gemericum basement, Western Carpathians, Slovakia: U-Pb (SHRIMP) data from zircons. Int J Earth Sci 99: 17-37 Vozárová A, Rodionov N, ŠArinová K, Presnyakov S (2017) New zircon ages on the Cambrian-Ordovician volcanism of the Southern Gemericum basement (Western Carpathians, Slovakia): SHRIMP dating, geochemistry and provenance. Int J Earth Sci 106: 2147-2170 